\title{
DISCRETE QUASICONFORMAL GROUPS I
}

\author{
F. W. GEHRING and G. J. MARTIN \\ [Received 6 January 1986-Revised 27 January 1986]
}

\section{Introduction}

In this paper we begin an investigation into the geometry of discrete quasiconformal groups. The structure of general topological groups is not as well understood as that of conformal or Möbius groups, a class of groups which have been studied intensively for many years. We hope to gain additional insight into both families by studying groups of homeomorphisms of uniformly bounded dilatation acting on a domain $D$ in $\overline{\mathbb{R}}^{n}$, a class which lies between the two kinds mentioned above.

One natural way to construct such a group is to conjugate a conformal group by a quasiconformal mapping. Gehring and Palka first asked in [9] if this were the only method, that is, if each quasiconformal group is the quasiconformal conjugate of a conformal group. Sullivan [27] and Tukia [28] answered this question in the affirmative for quasiconformal groups acting on subsets of the extended complex plane $\overline{\mathbb{R}}^{2}$; a recent paper by Hinkkanen [11] establishes an analogous result for quasisymmetric or one-dimensional quasiconformal groups acting on $\mathbb{R}$. Later Tukia [29] constructed for every $n>2$ a quasiconformal group which is not isomorphic as a topological group, and hence not quasiconformally conjugate, to a Möbius group. Tukia's examples are not discrete groups and his methods were subsequently modified by Martin [21] to yield discrete groups which are not quasiconformally conjugate to a conformal group. Finally McKemie [22] used Tukia's methods to construct for each $K>1$ a $K$ quasiconformal group not quasiconformally conjugate to a Möbius group.

We restrict our attention in this article to discrete quasiconformal groups acting on $\overline{\mathbb{R}}^{n}$ and show that these groups resemble in many ways their conformal counterparts. In $\S 3$ we introduce a characteristic convergence property for quasiconformal groups and devote the remainder of Part I of this paper to the study of convergence groups, groups of self homeomorphisms of $\mathbb{\mathbb { R }}^{n}$ which have this property. In $\S 4$ we derive several important properties of the limit set $L(G)$ of a discrete convergence group $G$; for example, $L(G)$ is perfect if it contains more than two points. In $\$ 5$ we describe the elementary discrete convergence groups $G$, that is, those with $\operatorname{card}(L(G)) \leqslant 2$, and show that we can classify the elements $g$ of a discrete convergence group $G$ as elliptic, parabolic, or loxodromic according to their order and number of fixed points. In $\S 6$ we study the behaviour of iterates of parabolic and loxodromic elements and then use this information to investigate the structure and limit sets of the non-elementary discrete convergence groups $G$ with $\operatorname{card}(L(G))>2$. Finally, in $\S 7$ we derive a condition sufficient to imply that a group of homeomorphisms is a convergence

The research of the first author was supported in part by the Science Research Council of the United Kingdom, while he was visiting the University of Cambridge, and by the U.S. National Science Foundation, and that of the second author was supported in part by the Sloan Foundation. 
group, and give examples to show that a convergence group need not be topologically conjugate to a Möbius group.

Part II of this article will be published separately. There we will employ distortion properties of quasiconformal mappings to derive more detailed results concerning the structure of discrete quasiconformal groups. We will also study the Poincaré series for the groups which map a ball or half space onto itself.

\section{Notation and definitions}

For $n \geqslant 2$ we let $\mathbb{R}^{n}$ denote euclidean $n$-space, $\overline{\mathbb{R}}^{n}$ its one point compactification $\mathbb{R}^{n} \cup\{\infty\}$, and $e_{1}, \ldots, e_{n}$ the standard orthonormal basis for $\mathbb{R}^{n}$.

A Möbius transformation acting on $\overline{\mathbb{R}}^{n}$ is a finite composition of reflections in spheres and hyperplanes; we let $\mathrm{GM}\left(\overline{\mathbb{R}}^{n}\right)$ denote the group of all such transformations.

Stereographic projection $p$ is the mapping from $\overline{\mathbb{R}}^{n}$ onto the unit sphere $S^{n}$ in $\mathbb{R}^{n+1}$ given by

$$
p(x)=e_{n+1}+\frac{2\left(x-e_{n+1}\right)}{\left|x-e_{n+1}\right|^{2}} .
$$

We define the chordal distance between two points $x$ and $y$ in $\overline{\mathbb{R}}^{n}$ as

$$
q(x, y)=|p(x)-p(y)|,
$$

and let $B_{q}(x, r)$ denote the chordal ball

$$
B_{q}(x, r)=\left\{y \in \overline{\mathbb{R}}^{n}: q(x, y)<r\right\} .
$$

Throughout this paper, the topology of $\overline{\mathbb{R}}^{n}$ and all notions of convergence will be taken with respect to the chordal metric.

2.1. Groups of homeomorphisms. Suppose that $G$ is a group of self homeomorphisms of a domain $D$ in $\overline{\mathbb{R}}^{n}$. Then $G$ is a $K$-quasiconformal group if each $g \in G$ is $K$-quasiconformal in $D ; G$ is a quasiconformal group if it is $K$ quasiconformal for some $K$. By the general form of Liouville's theorem, a 1-quasiconformal group is in fact the restriction to $D$ of a Möbius group when $n \geqslant 3$. See, for example, $[8,26,4]$.

Next we say that $G$ is discrete if it contains no infinite sequence of distinct elements which converge uniformly on compact subsets, or simply $c$-uniformly, in $D$ to an element of $G$.

The group $G$ is said to be discontinuous at a point $x \in D$ if there is a neighbourhood $U$ of $x$ in $D$ such that

$$
g(U) \cap U=\varnothing
$$

for all but finitely many $g \in G$. We denote by $O(G)$ the set of all $x \in D$ at which $G$ is discontinuous and call $L(G)=\bar{D} \backslash O(G)$ the limit set of $G$; we say that $G$ is discontinuous if $O(G)$ is not empty. It is easy to check that a discontinuous group is discrete; the converse is not true [2].

We say that $G$ is properly discontinuous in an open set $O \subset D$ if for each compact set $F \subset O$,

$$
g(F) \cap F=\varnothing
$$


for all but finitely many $g \in G$. In this case it follows that $G$ must be discontinuous at each point of $O$ and that

$$
L(G) \subset \tilde{D} \backslash O
$$

By a sequence we will always mean an infinite sequence of distinct elements of $G$. Finally, we say that a set $E \subset D$ is $G$-invariant if $g(E)=E$ for each $g \in G$. The following result is then an immediate consequence of the definitions given above.

2.4. Lemma. The set $O(G)$ is an open set and $L(G)$ is a closed set; they are both $G$-invariant.

\section{Compactness}

Möbius transformations and quasiconformal mappings share certain convergence properties. We give one of these which has far-reaching implications.

3.1. Lemma. Suppose that $D$ is a domain in $\overline{\mathbb{R}}^{n}$ and that $F$ is a family of $K$-quasiconformal mappings of $D$ which is not equicontinuous in $D$. Then there exist a point $x_{0}$ in $D$ and an infinite subfamily $F_{0}$ of $F$ which is equicontinuous in $D_{0}=D \backslash\left\{x_{0}\right\}$.

Proof. Since $F$ is not equicontinuous in $D$, there exist a point $x_{0} \in D$ and a positive constant $a$ such that for $j=1,2, \ldots$ we can find a mapping $f_{j} \in F$ and a point $x_{j} \in D \backslash\left\{x_{0}\right\}$ with $q\left(x_{j}, x_{0}\right)<1 / j$ and

$$
q\left(f_{j}\left(x_{j}\right), f_{j}\left(x_{0}\right)\right) \geqslant a>0 .
$$

Let $F_{0}=\left\{f_{j}\right\}$ and for $k=1,2, \ldots$ set

$$
\begin{aligned}
D_{k} & =D \backslash\left\{x_{0}, x_{k}, x_{k+1}, \ldots\right\}, \\
b_{k} & =\min _{1 \leqslant j \leqslant k} q\left(f_{j}\left(x_{k}\right), f_{j}\left(x_{0}\right)\right)>0 .
\end{aligned}
$$

Then for each $j$ and $k, \mathbb{R} \backslash f_{j}\left(D_{k}\right)$ contains a pair of points whose chordal distance from one another is at least $\min \left(a, b_{k}\right)>0$. Thus the family $F_{0}$ is equicontinuous in each domain $D_{k}$, and hence in their union $D_{0}$, by Theorem 19.2 in [31].

The following consequence of Lemma 3.1 plays a crucial role in what follows.

3.2. TheOrem. Suppose that $F$ is an infinite family of $K$-quasiconformal self mappings of $\tilde{\mathbb{R}}^{n}$. Then there exists a sequence $\left\{f_{j}\right\}$ in $F$ such that one of the following is true.

A. There exists a K-quasiconformal self mapping $f$ of $\overline{\mathbb{R}}^{n}$ such that

$$
\lim _{j \rightarrow \infty} f_{j}=f \quad \text { and } \quad \lim _{j \rightarrow \infty} f_{j}^{-1}=f^{-1}
$$

uniformly in $\overline{\mathbb{R}}^{n}$.

B. There exist points $x_{0}, y_{0}$ in $\overline{\mathbb{R}}^{n}$ such that

$$
\lim _{j \rightarrow \infty} f_{j}=y_{0} \quad \text { and } \quad \lim _{j \rightarrow \infty} f_{j}^{-1}=x_{0}
$$


c-uniformly in $\overline{\mathbb{R}}^{n} \backslash\left\{x_{0}\right\}$ and $\overline{\mathbb{R}}^{n} \backslash\left\{y_{0}\right\}$, respectively. The possibility that $x_{0}=y_{0}$ may occur.

Proof. Suppose that $F$ has an infinite subfamily $F_{0}$ which is equicontinuous in $\overline{\mathbb{R}}^{n}$. By Ascoli's theorem there is a subsequence $\left\{f_{j}\right\}$ of mappings in $F_{0}$ which converge to a limit mapping $f$ uniformly in $\overline{\mathbb{R}}^{n}$. Then it follows from Theorems $21.1,37.2$, and 21.11 of [31] that $f$ is a $K$-quasiconformal self mapping of $\overline{\mathbb{R}}^{n}$ and that $f_{j}^{-1}$ converges to $f^{-1}$ uniformly in $\overline{\mathbb{R}}^{n}$. In particular, we note that $G_{0}=\left\{f_{j}^{-1}\right\}$ is an infinite subfamily of $G=\left\{f^{-1}: f \in F\right\}$ which is equicontinuous in $\mathbb{\mathbb { R }}^{n}$.

Suppose now that $F$ contains no infinite subfamily which is equicontinuous in $\overline{\mathbb{R}}^{n}$. Then by Lemma 3.1 , there exist a point $x_{0}$ and an infinite subfamily $F_{0}$ which is equicontinuous in $\mathbb{\mathbb { R }}^{n} \backslash\left\{x_{0}\right\}$; as before we can extract a subsequence $\left\{f_{j}\right\}$ which converges $c$-uniformly in $\overline{\mathbb{B}}^{n} \backslash\left\{x_{0}\right\}$ to a mapping $f$. From Theorems 21.1 and 37.2 in [31] it follows that $f$ is either a $K$-quasiconformal mapping of $\overline{\mathbb{R}}^{n} \backslash\left\{x_{0}\right\}$ or a constant. The first possibility would imply that $f$ has a quasiconformal extension to $\overline{\mathbb{R}}^{n}$, that $f \rightarrow f_{j}$ uniformly in $\overline{\mathbb{R}}^{n}$, and hence that $\left\{f_{j}\right\}$ is an infinite subfamily of $F$ which is equicontinuous in $\overline{\mathbb{R}}^{n}$, contradicting our hypothesis. Thus $f$ must be equal to some point $y_{0} \in \overline{\mathbb{R}}^{n}$.

Next it follows from what was proved in the first part that the family $G=\left\{f^{-1}: f \in F\right\}$ does not contain an infinite subfamily which is equicontinuous in $\overline{\mathbb{R}}^{n}$. Hence we can argue as above to find points $x_{1}, y_{1} \in \mathbb{R}^{n}$ and a subsequence of $\left\{f_{j}^{-1}\right\}$ which converges to $y_{1} c$-uniformly in $\mathbb{\mathbb { R }}^{n} \backslash\left\{x_{1}\right\}$; by relabelling we may assume that $f_{j}^{-1} \rightarrow y_{1} c$-uniformly in $\overline{\mathbb{R}}^{n} \backslash\left\{x_{1}\right\}$. Then $\left\{f_{j}\right\}$ will be the desired sequence if we can show that $y_{0}=x_{1}$ and $x_{0}=y_{1}$.

Suppose that $y_{0} \neq x_{1}$ and choose $x \in \overline{\mathbb{R}}^{n} \backslash\left\{x_{0}, y_{1}\right\}$. Then $f_{j}(x) \rightarrow y_{0}$ and so for large $j$ the points $f_{j}(x)$ are compactly contained in $\mathbb{\mathbb { R }}^{n} \backslash\left\{x_{1}\right\}$. Hence

$$
x=\lim _{j \rightarrow \infty} f_{j}^{-1}\left(f_{j}(x)\right)=y_{1}
$$

and we have a contradiction. Thus $y_{0}=x_{1}$. A similar procedure yields $x_{0}=y_{1}$ and the proof is complete.

The conclusions of Theorem 3.2 motivate the following definition which will allow us to see to what extent the above convergence properties determine the geometric behaviour of Möbius and quasiconformal groups. (See also [19].)

3.3. Definition. A family $F$ of self homeomorphisms of $\mathbb{R}^{n}$ is said to have the convergence property if each infinite subfamily of $F$ contains a sequence $\left\{f_{j}\right\}$ such that one of the following is true.

A. There exists a self homeomorphism $f$ of $\overline{\mathbb{B}}^{n}$ such that

$$
\lim _{j \rightarrow \infty} f_{j}=f \text { and } \lim _{j \rightarrow \infty} f_{j}^{-1}=f^{-1}
$$

uniformly in $\overline{\mathbb{R}}^{n}$.

B. There exist points $x_{0}, y_{0}$ in $\overline{\mathbb{R}}^{n}$ such that

$$
\lim _{j \rightarrow \infty} f_{j}=y_{0} \quad \text { and } \quad \lim _{j \rightarrow \infty} f_{j}^{-1}=x_{0}
$$

$c$-uniformly in $\overline{\mathbb{R}}^{n} \backslash\left\{x_{0}\right\}$ and $\overline{\mathbb{R}}^{n} \backslash\left\{y_{0}\right\}$, respectively. 
3.4. Definition. A group $G$ of self homeomorphisms of $\overline{\mathbb{R}}^{n}$ is said to be a convergence group if it has the convergence property.

3.5. COROllaRY. If $G$ is a quasiconformal group acting on $\mathbb{\mathbb { R }}^{n}$, then $G$ is a convergence group. In particular, every Möbius group is a convergence group.

3.6. REMARK. If $f$ is a self homeomorphism of $\overline{\mathbb{R}}^{n}$ and if $G$ is a convergence group, then $f \circ G \circ f^{-1}$ is also a convergence group. Hence there exist convergence groups which are neither Möbius nor quasiconformal groups. Moreover, Tukia's example in [29] is a quasiconformal group which is not isomorphic, and hence not topologically conjugate, to a Möbius group. See $\$ 7$ for further examples.

The convergence property takes the following form for discrete groups.

3.7. THEOREM. If $G$ is a discrete convergence group, then for each infinite sequence of elements in $G$ there exist a subsequence $\left\{g_{j}\right\}$ and points $x_{0}, y_{0}$ in $\overline{\mathbb{B}}^{n}$ such that

$$
\lim _{j \rightarrow \infty} g_{j}=y_{0} \quad \text { and } \quad \lim _{j \rightarrow \infty} g_{j}^{-1}=x_{0}
$$

c-uniformly in $\tilde{\mathbb{R}}^{n} \backslash\left\{x_{0}\right\}$ and $\overline{\mathbb{R}}^{n} \backslash\left\{y_{0}\right\}$, respectively. Moreover, $x_{0}$, y $y_{0}$ lie in each $G$-invariant closed set $E$ which contains at least two points.

Proof. Because $G$ has the convergence property, there exists a sequence $\left\{g_{j}\right\}$ in $G$ such that $g_{j} \rightarrow g$ and $g_{j}^{-1} \rightarrow g^{-1}$ uniformly in $\overline{\mathbb{R}}^{n}$, where $g$ is a self homeomorphism of $\overline{\mathbb{R}}^{n}$, or such that $g_{j} \rightarrow y_{0}$ and $g_{j}^{-1} \rightarrow x_{0} c$-uniformly in $\overline{\mathbb{R}}^{n} \backslash\left\{x_{0}\right\}$ and $\overline{\mathbb{R}}^{n} \backslash\left\{y_{0}\right\}$, respectively, where $x_{0}, y_{0} \in \overline{\mathbb{R}}^{n}$. The first case cannot occur, since otherwise $\left\{g_{j+1} \circ g_{j}^{-1}\right\}$ would contain an infinite number of distinct elements of $G$ which converge uniformly in $\overline{\mathbb{R}}^{n}$ to the identity of $G$.

Suppose next that $E$ is a $G$-invariant closed set with $\operatorname{card}(E) \geqslant 2$ and choose $x \in E \backslash\left\{x_{0}\right\}$. Then the points $g_{j}(x)$ must lie in $E$ and hence so must their limit $y_{0}$. A similar argument shows that $x_{0} \in E$ and the proof is complete.

The following immediate consequence of Theorem 3.7 further relates the notions of discreteness and discontinuity for convergence groups.

3.8. Corollary. Suppose that $G$ is a discrete convergence group and that $E$ is a $G$-invariant closed proper subset of $\mathbb{R}^{n}$ which contains at least two points. Then $G$ is properly discontinuous in, and hence discontinuous at each point of, $\overline{\mathbb{R}}^{n} \backslash E$. In particular, $L(G) \subset E$.

Proof. Suppose that $G$ is not properly discontinuous in $\overline{\mathbb{R}}^{n} \backslash E$. Then there exist a compact subset $F$ of $\mathbb{\mathbb { R }}^{n} \backslash E$ and a sequence $\left\{g_{j}\right\}$ in $G$ such that

$$
g_{j}(F) \cap F \neq \varnothing
$$

for all $j$. By Theorem 3.7 there exist a subsequence, which we again denote by $\left\{g_{j}\right\}$, and points $x_{0}, y_{0} \in E$ such that $g_{j} \rightarrow y_{0}$ uniformly in $F$. Let $V$ be a neighbourhood of $y_{0}$ such that $V \cap F=\varnothing$. Then $g_{j}(F) \subset V$ for large $j$ and we 
obtain a $g_{j}$ for which (3.9) cannot hold. Thus $G$ must be properly discontinuous in $\overline{\mathbb{R}}^{n} \backslash E$.

3.10. Corollary. Suppose that $G$ is a discrete convergence group and that $E_{1}$, $E_{2}, E_{3}$ are pairwise disjoint closed sets in $\overline{\mathbb{R}}^{n}$. Then at most finitely many $g$ in $G$ satisfy

$$
g\left(E_{k}\right) \cap E_{k} \neq \varnothing \text { for } k=1,2,3
$$

Proof. If infinitely many $g \in G$ satisfy (3.11), then by Theorem 3.7 we can choose a sequence $\left\{g_{j}\right\}$ in $G$ and points $x_{0}, y_{0}$ such that $g_{j} \rightarrow y_{0} c$-uniformly in $\overline{\mathbb{R}}^{n} \backslash\left\{x_{0}\right\}$ and such that

$$
g_{j}\left(E_{k}\right) \cap E_{k} \neq \varnothing
$$

for all $j$ and $k$. By relabelling if necessary, we may assume that $E_{1} \cup E_{2} \subset$ $\overline{\mathbb{R}}^{n} \backslash\left\{x_{0}\right\}$. Then $g_{j} \rightarrow y_{0}$ uniformly in $E_{1} \cup E_{2}$ and with (3.12) we obtain

$$
q\left(y_{0}, E_{k}\right) \leqslant \lim _{j \rightarrow \infty} q\left(y_{0}, g_{j}\left(E_{k}\right)\right)=0 \text {, }
$$

whence $y_{0} \in E_{k}$ for $k=1,2$, a contradiction.

3.13. Definition. We denote by $\operatorname{fix}(G)$ the set of points in $\overline{\mathbb{R}}^{n}$ which are fixed by each element in $G$.

3.14. Corollary. If $G$ is an infinite discrete convergence group, then $\operatorname{card}(\operatorname{fix}(G)) \leqslant 2$.

We consider next to what extent the family of $K$-quasiconformal mappings is maximal with regard to the convergence property in Definition 3.3. Cf. [3] and [8, Theorem 18].

3.15. Definition. A family $F$ of self homeomorphisms of $\overline{\mathbb{R}}^{n}$ is said to be complete with respect to Möbius transformations if for each $g, h$ in $\mathrm{GM}\left(\overline{\mathbb{R}}^{n}\right)$ the mapping $g \circ f \circ h$ lies in $F$ whenever $f$ does.

3.16. THEOREM. Let $F$ be a family of self homeomorphisms of $\overline{\mathbb{R}}^{n}$ which is complete with respect to Möbius transformations. Then $F$ has the convergence property if and only if there is a constant $K$ such that each $f$ in $F$ is $K$-quasiconformal.

Proof. The sufficiency follows from Theorem 3.2. For the necessity let

$$
K=\sup \max _{|x|=1}|f(x)|
$$

where the supremum is taken over all $f \in F$ which fix the points $0, e_{1}, \infty$. Then there is a sequence of mappings $\left\{f_{i}\right\}$ in $F$ normalized as above for which

$$
K=\lim _{j \rightarrow \infty} \max _{|x|=1}\left|f_{j}(x)\right| .
$$

Since $F$ has the convergence property and since the mappings $f_{j}$ fix three points, 
there exists a subsequence which converges to a homeomorphism uniformly in $\overline{\mathbb{R}}^{n}$. Hence $K$ is finite.

Next let $f$ be any mapping in $F$. We wish to show that $f$ is $K$-quasiconformal. Since $F$ is closed under conjugation by Möbius transformations, we may assume, without loss of generality, that $f$ also fixes $\infty$. Fix $x_{0} \in \mathbb{R}^{n}$ and $r>0$, and choose $x_{1}$ such that $\left|x_{1}-x_{0}\right|=r$ and

$$
\left|f\left(x_{1}\right)-f\left(x_{0}\right)\right|=m_{f}\left(x_{0}, r\right)=\min _{\left|x-x_{0}\right|=r}\left|f(x)-f\left(x_{0}\right)\right| .
$$

Let $g$ and $h$ be similarity mappings such that

$$
h\left(e_{1}\right)=x_{1}, \quad h(0)=x_{0}, \quad g\left(f\left(x_{1}\right)\right)=e_{1}, \quad g\left(f\left(x_{0}\right)\right)=0 .
$$

Then $g \circ f \circ h$ is a normalized element of $F$,

$$
\begin{aligned}
M_{f}\left(x_{0}, r\right) & =\max _{\left|x-x_{0}\right|=r}\left|f(x)-f\left(x_{0}\right)\right| \\
& =m_{f}\left(x_{0}, r\right) \max _{|x|=1}|g \circ f \circ h(x)| \leqslant K m_{f}\left(x_{0}, r\right),
\end{aligned}
$$

and $f$ is quasiconformal with linear dilatation bounded by $K$.

3.17. Corollary. A self homeomorphism $f$ of $\mathbb{R}^{n}$ is quasiconformal if and only if the family

$$
F=\left\{g \circ f \circ h: g, h \in \mathrm{GM}\left(\overline{\mathbb{R}}^{n}\right)\right\}
$$

has the convergence property.

Tukia and Väisälä have shown [30] that if $G$ is a quasiconformal group acting on $\mathbb{R}^{n}$ which contains the group of all similarity mappings $\mathrm{GM}\left(\mathbb{R}^{n}\right)$, then $G$ is precisely the group $\operatorname{GM}\left(\mathbb{R}^{n}\right)$. If we combine this fact with what was proved above, we obtain the following result.

3.18. THEOREM. If $G$ is a convergence group which contains a subgroup which is dense in $G M\left(\overline{\mathbb{R}}^{n}\right)$, then $G$ is a subgroup of $\mathrm{GM}\left(\overline{\mathbb{R}}^{n}\right)$.

Proof. Let $H$ denote the family of self homeomorphisms $h$ of $\overline{\mathbb{R}}^{n}$ for which there exists a sequence $\left\{g_{j}\right\}$ in $G$ such that $g_{j} \rightarrow h$ and $g_{j}^{-1} \rightarrow h^{-1}$ uniformly in $\overline{\mathbb{R}}^{n}$. Then $H$ is a convergence group which contains $\operatorname{GM}\left(\overline{\mathbb{R}}^{n}\right)$ and hence a quasiconformal group by Theorem 3.16 .

Let $H_{0}=\{h \in H: h(0)=0\}$ and let $f$ be a Möbius transformation for which $f(0)=\infty$. Then $f \circ H_{0} \circ f^{-1}$ is a quasiconformal group acting on $\mathbb{R}^{n}$ which contains $\mathrm{GM}\left(\mathbb{R}^{n}\right)$. Thus

$$
H_{0}=f^{-1} \circ \mathrm{GM}\left(\mathbb{R}^{n}\right) \circ f \subset \mathrm{GM}\left(\overline{\mathbb{R}}^{n}\right)
$$

by the aforementioned result.

Finally, given any $g \in G$ we can find a Möbius transformation $f$ such that $f \circ g$ is in $H_{0}$. Hence $g$ is a Möbius transformation.

Theorem 3.16 shows that the convergence property essentially characterizes quasiconformal groups which act on $\overline{\mathbb{R}}^{n}$. We can formulate this property for any 
domain $D$ by replacing $\overline{\mathbb{R}}^{n}$ by $D$ and 'uniformly' by ' $c$-uniformly' in Definition 3.3. We conclude this section by observing, however, that an infinite group which satisfies the convergence property on a proper subdomain of $\overline{\mathbb{R}}^{n}$ cannot be discrete.

3.19. THEOREM. Suppose that $D$ is a domain in $\overline{\mathbb{R}}^{n}$ and that $G$ is a group of self homeomorphisms of $D$ which has the convergence property on $D$. If $G$ is infinite and discrete, then $D=\overline{\mathbb{R}}^{n}$.

Proof. As in the proof of Theorem 3.7 there exist points $x_{0}, y_{0} \in D$ and a sequence $\left\{g_{j}\right\}$ in $G$ such that $g_{j} \rightarrow y_{0}$ and $g_{j}^{-1} \rightarrow x_{0} c$-uniformly in $D \backslash\left\{x_{0}\right\}$ and $D \backslash\left\{y_{0}\right\}$, respectively. Let $U, V$ be chordal balls about $x_{0}, y_{0}$, respectively, with closures in $D$. By the $c$-uniform convergence there exists an integer $j$ such that $g_{j}(\partial U) \subset V$ and $g_{j}^{-1}(\partial V) \subset U$ whence

$$
\partial g_{j}(U) \cap(D \backslash V)=\varnothing \quad \text { and } \quad g_{j}(U) \cap(D \backslash V) \neq \varnothing .
$$

Since $D \backslash V$ is connected, (3.20) implies that $D \backslash V \subset g_{j}(U)$ and hence that

$$
\bar{D} \subset g_{j}(\bar{U}) \cup \bar{V} \subset D .
$$

Thus $D=\overline{\mathbb{R}}^{n}$.

\section{Limit set of a discrete convergence group}

We assume throughout this section that $G$ is a discrete convergence group acting on $\mathbb{R}^{n}$. We begin with a result which shows that each point of $L(G)$ is the limit under elements of $G$ of points of $O(G)$.

4.1. Lemma. For each point $y_{1}$ in $L(G)$ there exist a point $x_{1}$ in $L(G)$ and a sequence $\left\{g_{j}\right\}$ in $G$ such that

$$
\lim _{j \rightarrow \infty} g_{j}=y_{1} \text { and } \lim _{j \rightarrow \infty} g_{j}^{-1}=x_{1}
$$

c-uniformly in $\overline{\mathbb{R}}^{n} \backslash\left\{x_{1}\right\}$ and $\overline{\mathbb{R}}^{n} \backslash\left\{y_{1}\right\}$, respectively.

Proof. For each $j=1,2, \ldots$, let $V_{j}=B_{q}\left(y_{1}, r_{j}\right)$ where the $r_{j}$ are positive and converge to 0 . Since $G$ is not discontinuous at $y_{1}$, we can choose a sequence $\left\{h_{j}\right\}$ in $G$ such that

$$
h_{j}\left(V_{j}\right) \cap V_{j} \neq \varnothing
$$

for all $j$. Next by Theorem 3.7 we can choose a subsequence, which we again label $\left\{h_{j}\right\}$, and points $x_{0}, y_{0} \in \overline{\mathbb{R}}^{n}$ such that $h_{j} \rightarrow y_{0}$ and $h_{j}^{-1} \rightarrow x_{0} c$-uniformly in $\overline{\mathbb{R}}^{n} \backslash\left\{x_{0}\right\}$ and $\overline{\mathbb{R}}^{n} \backslash\left\{y_{0}\right\}$, respectively.

Suppose that $y_{1}=x_{0}$. Then we get the desired conclusion by taking $x_{1}=y_{0}$ and $g_{j}=h_{j}^{-1}$. Suppose next that $y_{1} \neq x_{0}$. By (4.2) we can find for each $j$ a point $z_{j} \in V_{j}$ with $h_{j}\left(z_{j}\right) \in V_{j}$. These points are compactly contained in $\overline{\mathbb{R}}^{n} \backslash\left\{x_{0}\right\}$ for large $j$ and $h_{j}\left(z_{j}\right) \rightarrow y_{0}$ by the $c$-uniform convergence. Thus

$$
q\left(y_{1}, y_{0}\right) \leqslant \lim _{j \rightarrow \infty}\left(q\left(y_{1}, h_{j}\left(z_{j}\right)\right)+q\left(h_{j}\left(z_{j}\right), y_{0}\right)\right)=0
$$

$y_{1}=y_{0}$, and we may take $x_{1}=x_{0}$ and $g_{j}=h_{j}$. 
If $U$ is a neighbourhood of $x_{1}$ and if $x \in U \backslash\left\{y_{1}\right\}$, then $g_{j}^{-1}(x) \rightarrow x_{1}$,

$$
g_{j}^{-1}(x) \in g_{j}^{-1}(U) \cap U \neq \varnothing
$$

for large $j$, and $G$ is not discontinuous at $x_{1}$. Hence $x_{1} \in L(G)$.

4.3. Definition. For each $x$ in $\overline{\mathbb{R}}^{n}$ we let $L(x, G)$ denote the set of all $y$ in $\overline{\mathbb{R}}^{n}$ for which there exists a sequence $\left\{g_{j}\right\}$ in $G$ such that $g_{j}(x) \rightarrow y$.

4.4. Lemma. We have $L(x, G) \subset L(G)$ for all $x$ in $\overline{\mathbb{R}}^{n}$ with equality whenever $x$ is not fixed by $G$.

Proof. Let $U$ be a neighbourhood of a point $y_{1} \in L(x, G)$, choose a sequence $\left\{g_{j}\right\}$ in $G$ such that $g_{j}(x) \in U$ for all $j$, and let $h_{j}=g_{j} \circ g_{1}^{-1}$. Then $\left\{h_{j}\right\}$ is a sequence in $G$ and

$$
g_{j}(x) \in h_{j}(U) \cap U \neq \varnothing
$$

for all $j$. Hence $G$ is not discontinuous at $y_{1}$ and $y_{1} \in L(G)$.

Suppose next that $y_{1} \in L(G)$ and let $x_{1}$ and $\left\{g_{i}\right\}$ be as in Lemma 4.1. If $x$ is not fixed by $G$, there exists $g \in G$ such that $x \neq x_{1}$ or $g(x) \neq x_{1}$. Hence either $g_{j}(x) \rightarrow y_{1}$ or $g_{j} \circ g(x) \rightarrow y_{1}$ and we conclude that $y_{1} \in L(x, G)$.

4.5. ThEOREM. If $L(G)$ contains three points, then $L(G)$ is a perfect set.

Proof. Let $y_{1} \in L(G)$ and choose $x_{1}$ and $\left\{g_{j}\right\}$ as in Lemma 4.1. Since $\operatorname{card}(L(G)) \geqslant 3$, there exists an $x_{2} \in L(G) \backslash\left\{x_{1}, y_{1}\right\}$.

If $g_{j}\left(x_{2}\right) \neq y_{1}$ for infinitely many $j$, then $\left\{g_{j}\left(x_{2}\right)\right\}$ is an infinite subset of $L(G)$ which has $y_{1}$ as a limit point. Otherwise we may assume that

$$
g_{j}\left(x_{2}\right)=y_{1}
$$

for all $j$. If $y_{1} \neq x_{1}$, then by (4.6), $\left\{g_{j}\left(y_{1}\right)\right\}$ is an infinite sequence of points in $L(G)$ which converges to $y_{1}$. Next if $y_{1}=x_{1}$, then there exists a point

$$
x_{3} \in L(G) \backslash\left\{x_{1}, x_{2}\right\},
$$

and $\left\{g_{j}\left(x_{3}\right)\right\}$ is again an infinite subset of $L(G)$ with limit point $y_{1}$. Thus $y_{1}$ is a limit point of $L(G)$ and so $L(G)$ is perfect.

The dichotomy that $L(G)$ contain fewer than three points or be perfect is a classical result for Möbius groups and motivates the following definition.

4.7. Definition. We say that $G$ is an elementary group if the cardinality of $L(G)$ is at most 2. Otherwise we say that $G$ is non-elementary.

We conclude this section with two further results on the limit set which depend on results established in $\$ \S 5$ and 6 . The first shows that the notions of discontinuous and properly discontinuous coincide for convergence groups.

4.8. THEOREM. If $G$ is discontinuous, then $G$ is properly discontinuous in $\overline{\mathbb{R}}^{n} \backslash L(G)$.

Proof. By Lemma 2.4 and hypothesis, $L(G)$ is a $G$-invariant closed proper subset of $\overline{\mathbb{R}}^{n}$. Hence Corollary 3.8 implies that $G$ is properly discontinuous in $\overline{\mathbb{R}}^{n} \backslash L(G)$ if $L(G)$ contains at least two points. 
If $L(G)=\left\{x_{0}\right\}$ and if $F$ is any compact set in $\overline{\mathbb{R}}^{n} \backslash L(G)$, then Theorem 5.10 and Lemma 5.5, with $E_{1}=\left\{x_{0}\right\}$ and $E_{2}=F$, imply that $g(F) \cap F=\varnothing$ for all but a finite number of $g \in G$.

Finally, if $L(G)=\varnothing$, then $G$ is finite by Theorem 5.7 and there is nothing to prove.

4.9. THEOREM. The set $L(G)$ is either nowhere dense or coincides with $\overline{\mathbb{R}}^{n}$.

Proof. We may assume that $G$ is non-elementary and hence that $L(G)$ is a non-empty perfect set. Suppose that $L(G) \neq \overline{\mathbb{R}}^{n}$. Then there exists a point $x \in \partial L(G)$. Let $V$ denote any neighbourhood of a point $y \in L(G)$. Then $y \in L(x, G)$ by Theorem 6.10 and there exists a $g \in G$ such that $g(x) \in V$. Hence $g^{-1}(V)$ is a neighbourhood of $x$ which contains a point $x_{0} \in O(G)$, and $y_{0}=g\left(x_{0}\right)$ lies in $V \backslash L(G)$. Thus $\operatorname{int}(L(G))=\varnothing$ and $L(G)$ is nowhere dense.

\section{The elementary discrete convergence groups}

We assume again throughout this section that $G$ is a discrete convergence group. We begin by classifying the elements of $G$ exactly as in the case of a discrete Möbius group. We then study the structure of the elementary groups and use this information to show that our classification of elements is exhaustive.

5.1. Definition. For each $g$ in $G$ we let

$$
\operatorname{ord}(g)=\inf \left\{m>0: g^{m}=\text { identity }\right\}, \quad \text { fix }(g)=\left\{x \in \overline{\mathbb{R}}^{n}: g(x)=x\right\} .
$$

We say that $g$ is elliptic if $\operatorname{ord}(g)<\infty$, that $g$ is parabolic if $\operatorname{ord}(g)=\infty$ and $\operatorname{card}(\operatorname{fix}(g))=1$, and that $g$ is loxodromic if $\operatorname{ord}(g)=\infty$ and $\operatorname{card}(\operatorname{fix}(g))=2$.

The following partially motivates the above classification.

5.2. Lemma. If $g$ is in $G$ and $\operatorname{ord}(g)=\infty$, then

$$
\operatorname{card}(\operatorname{fix}(g)) \leqslant 2, \quad \text { fix }(g) \subset L(G) .
$$

Proof. Since ord $(g)=\infty$, the subgroup $\langle g\rangle$ generated by powers of $g$ is an infinite discrete convergence group. Hence

$$
\operatorname{card}(\operatorname{fix}(g))=\operatorname{card}(\operatorname{fix}(\langle g\rangle)) \leqslant 2
$$

by Corollary 3.14. Next if $x$ is fixed by $g$, then it is fixed by each element in $\langle g\rangle$. Hence $G$ cannot be discontinuous at $x$ and $x \in L(G)$.

We say that $U$ is a topological ball if $U$ is the image of an open euclidean ball under a self homeomorphism of $\overline{\mathbb{R}}^{n}$. We then have the following useful condition which implies that an element of $G$ is loxodromic.

5.3. Lemma. Suppose that $g$ is an element of $G$. If there exists a topological ball $U$ such that $g(\bar{U}) \subset U$, then $g$ is loxodromic with one fixed point in $U$ and the other in $\overline{\mathbb{B}}^{n} \backslash \bar{U}$. 
Proof. The element $g$ cannot be of finite order $m$ since otherwise we would have $\bar{U}=g^{m}(\bar{U}) \subset U$. Next the hypotheses imply that $g^{-1}(\tilde{V}) \subset V$ where $V=$ $\overline{\mathbb{R}}^{n} \backslash \bar{U}$. The Brouwer fixed point theorem [12] then implies that $g$ has a fixed point in $U$ and that $g^{-1}$, and hence $g$, has a fixed point in $V$. Thus $g$ has exactly two fixed points by Lemma 5.2 and hence is loxodromic.

5.4. Lemma. Suppose that $x_{0}, y_{0}$ are distinct points in $\overline{\mathbb{R}}^{n}$ and that $\left\{g_{j}\right\}$ is a sequence in $G$ which converges to $y_{0} c$-uniformly in $\overline{\mathbb{R}}^{n} \backslash\left\{x_{0}\right\}$. Then all but a finite number of the $g_{j}$ are loxodromic.

Proof. Let $U=B_{q}\left(y_{0}, r\right)$ where $0<r<q\left(x_{0}, y_{0}\right)$. Then $U$ is a chordal ball compactly contained in $\overline{\mathbb{R}}^{n} \backslash\left\{x_{0}\right\}$ and $g_{j}(\bar{U}) \subset U$ for all sufficiently large $j$ by virtue of the $c$-uniform convergence. Hence all but a finite number of the $g_{j}$ are loxodromic by Lemma 5.3 .

We have next the following sharpened form of Corollary 3.10.

5.5. LemMA. Suppose that $E_{1}, E_{2}$ are disjoint closed sets in $\mathbb{R}^{n}$. Then at most finitely many non-loxodromic $g$ in $G$ satisfy

$$
g\left(E_{k}\right) \cap E_{k} \neq \varnothing \text { for } k=1,2 .
$$

Proof. Suppose otherwise. Then by Theorem 3.7 we can find points $x_{0}, y_{0}$ and a sequence $\left\{g_{j}\right\}$ of non-loxodromic elements in $G$ such that $g_{j} \rightarrow y_{0} c$-uniformly in $\overline{\mathbb{R}}^{n} \backslash\left\{x_{0}\right\}$ and such that

$$
g_{j}\left(E_{k}\right) \cap E_{k} \neq \varnothing
$$

for all $j$ and $k$. If $E_{1} \cup E_{2} \subset \overline{\mathbb{R}}^{n} \backslash\left\{x_{0}\right\}$, then $g_{j} \rightarrow y_{0}$ uniformly in $E_{1} \cup E_{2}$ and (5.6) implies that $y_{0} \in E_{1} \cap E_{2}$, a contradiction. Hence we may assume that $x_{0} \in E_{1}$. Then $g_{j} \rightarrow y_{0}$ uniformly in $E_{2}$ and we have $y_{0} \in E_{2}$ by (5.6). Thus $x_{0} \neq y_{0}$ and all but a finite number of the $g_{j}$ are loxodromic by Lemma 5.4 .

We next classify the elementary groups in terms of the cardinality of their limit sets.

5.7. THEOREM. The set $L(G)$ is empty if and only if $G$ is a finite group of elliptic elements.

Proof. The sufficiency is trivial. For the necessity suppose that $G$ were infinite. Then by Theorem 3.7 there would exist points $x_{0}, y_{0}$ and a sequence $\left\{g_{j}\right\}$ in $G$ which converges to $y_{0} c$-uniformly in $\mathbb{R}^{n} \backslash\left\{x_{0}\right\}$; thus we would obtain

$$
y_{0} \in L(x, G) \subset L(G)
$$

by Lemma 4.4. Hence $G$ is finite with only elliptic elements.

Theorem 5.7 implies that each finite group of elliptic elements is elementary. It is natural to ask what one can say about arbitrary groups of elliptic elements.

5.8. LEMma. If $G$ contains no loxodromic elements, then either $G$ is finite or $L(G)$ consists of a single point which is fixed by each element in $G$. 
Proof. Suppose that $G$ is infinite. Then by Theorem 3.7 we can choose points $x_{0}, y_{0}$ and a sequence $\left\{g_{j}\right\}$ which converges to $y_{0} c$-uniformly in $\mathbb{R}^{n} \backslash\left\{x_{0}\right\}$. Since $G$ contains no loxodromic elements, Lemma 5.4 implies that $y_{0}=x_{0}$.

Next let $g$ be an element of $G$ and set $h_{j}=g_{j} \circ g^{-1}$. Then $h_{j} \rightarrow x_{0} c$-uniformly in $\overline{\mathbb{R}}^{n} \backslash\left\{g\left(x_{0}\right)\right\}$ and $g\left(x_{0}\right)=x_{0}$ again by Lemma 5.4 . Hence $x_{0}$ is fixed by the group $G$.

Finally, suppose that $y_{1}$ is a point of $L(G)$. Then by Lemma 4.1 there exist a point $x_{1}$ and a sequence $\left\{h_{j}\right\}$ in $G$ which converges to $y_{1} c$-uniformly in $\mathbb{R}^{n} \backslash\left\{x_{1}\right\}$. As above we see that $y_{1}=x_{1}$ and we conclude that $y_{1}=x_{0}$, since otherwise $x_{1} \neq x_{0}$ and

$$
x_{0}=\lim _{j \rightarrow \infty} h_{j}\left(x_{0}\right)=y_{1}
$$

Thus $L(G)=\left\{x_{0}\right\}$.

5.9. Corollary. If $G$ contains only elliptic elements, then $G$ is elementary and has at most one limit point.

5.10. Theorem. The set $L(G)$ contains exactly one point $x_{0}$ if and only if $G$ is an infinite group which contains only elliptic and parabolic elements which fix $x_{0}$.

Proof. If $L(G)=\left\{x_{0}\right\}$, then $G$ must be infinite and

$$
\left\{g\left(x_{0}\right)\right\}=g(L(G))=\left\{x_{0}\right\}
$$

for each $g \in G$ since $L(G)$ is $G$-invariant. Lemma 5.2 then implies that each element of $G$ is either of finite order or of infinite order with one fixed point. The sufficiency is an immediate consequence of Lemma 5.8 .

5.11. THEOREM. The set $L(G)$ contains exactly two points $x_{0}$ and $y_{0}$ if and only if $G$ is an infinite group which contains only loxodromic elements which fix $x_{0}$ and $y_{0}$, and elliptic elements which either fix or interchange $x_{0}$ and $y_{0}$. In addition, $G$ must contain at least one loxodromic element and at most finitely many elliptic elements which fix $x_{0}$ and $y_{0}$ or are of odd order.

Proof. Suppose that $L(G)=\left\{x_{0}, y_{0}\right\}$. Since $L(G)$ is $G$-invariant, each $g \in G$ satisfies one of the following conditions:

$$
\begin{array}{ll}
g\left(x_{0}\right)=x_{0}, & g\left(y_{0}\right)=y_{0} \\
g\left(x_{0}\right)=y_{0}, & g\left(y_{0}\right)=x_{0} .
\end{array}
$$

We show first that each $g$ which satisfies (5.13) is an elliptic element of even order. Suppose otherwise. Then

$$
\left\{x_{0}, y_{0}\right\}=\operatorname{fix}\left(g^{2}\right) \subset L\left(\left\langle g^{2}\right\rangle\right)
$$

by Lemma 5.2 , and by Lemma 4.1 there would exist a point $x_{1}$ and a sequence $\left\{h_{j}\right\}$ in $\left\langle g^{2}\right\rangle$ which converges to $y_{0} c$-uniformly in $\overline{\mathbb{R}}^{n} \backslash\left\{x_{1}\right\}$. Then for $x \in \overline{\mathbb{R}}^{n} \backslash\left\{x_{1}, g^{-1}\left(x_{1}\right)\right\}$ we would have

$$
y_{0}=\lim _{j \rightarrow \infty} h_{j}(g(x))=\lim _{j \rightarrow \infty} g\left(h_{j}(x)\right)=g\left(y_{0}\right)=x_{0},
$$

a contradiction. In particular, we see that $G$ does not contain parabolic elements since no such element $g$ could satisfy $(5.12)$. 
Since $\operatorname{card}(L(G))=2, G$ contains a loxodromic element by Lemma 5.8. Finally, Lemma 5.5 with $E_{1}=\left\{x_{0}\right\}$ and $E_{2}=\left\{y_{0}\right\}$ implies that $G$ has only finitely many elliptic elements which satisfy (5.12). In particular, $G$ contains only finitely many elliptic elements $g$ of odd order since no such $g$ can satisfy (5.13).

For the sufficiency $G$ contains by hypothesis a loxodromic element which fixes $x_{0}$ and $y_{0}$. Hence $\left\{x_{0}, y_{0}\right\} \subset L(G)$ by Lemma 5.2.

Suppose next that $y_{1} \in L(G)$. Then Lemma 4.1 yields a point $x_{1}$ and a sequence in $G$ which converges to $y_{1} c$-uniformly in $\overline{\mathbb{R}}^{n} \backslash\left\{x_{1}\right\}$. By hypothesis there exists a subsequence $\left\{g_{i}\right\}$ whose elements either all fix or all interchange the two points $x_{0}, y_{0}$. In either case,

$$
y_{1}=\left\{\begin{array}{lll}
\lim _{j \rightarrow \infty} g_{j}\left(x_{0}\right) \in\left\{x_{0}, y_{0}\right\} & \text { if } & x_{0} \neq x_{1}, \\
\lim _{j \rightarrow \infty} g_{j}\left(y_{0}\right) \in\left\{x_{0}, y_{0}\right\} & \text { if } & y_{0} \neq x_{1} .
\end{array}\right.
$$

Thus $L(G)=\left\{x_{0}, y_{0}\right\}$.

5.14. Remarks. We remind the reader that the classification given in Theorems 5.10 and 5.11 is for discrete convergence groups $G$.

The situations described in these results can take place. For example,

$$
g_{1}(z)=z+1, \quad g_{2}(z)=-z
$$

generate an infinite discrete convergence group $G$ which contains only elliptic and parabolic elements that fix $\infty$ and for which $L(G)=\{\infty\}$. Similarly, if $m$ is a prime, then

$$
g_{1}(z)=2 z, \quad g_{2}(z)=1 / z, \quad g_{3}(z)=e^{2 \pi i / m} z
$$

generate a discrete convergence group $G$ which contains infinitely many loxodromic elements which fix 0 and $\infty, m-1$ elliptic elements of order $m$ which fix 0 and $\infty$, and infinitely many elliptic elements of order 2 which interchange 0 and $\infty$. In addition, $L(G)=\{0, \infty\}$.

We show next that every element in a discrete convergence group is of one of the three types given in Definition 5.1. This fact is a consequence of the above description of the elementary groups and the following result.

5.15. THEOREM. If $G$ is an abelian group, then $G$ is elementary.

Proof. Suppose that $L(G)$ contains three points $y_{1}, y_{2}, y_{3}$. Then by Lemma 4.1 there are points $x_{1}, x_{2}, x_{3} \in L(G)$ and sequences $\left\{g_{1, j}\right\},\left\{g_{2, j}\right\},\left\{g_{3, j}\right\}$ in $G$ such that for $k=1,2,3, g_{k, j} \rightarrow y_{k} c$-uniformly in $\mathbb{R}^{n} \backslash\left\{x_{k}\right\}$. By symmetry and relabelling, we may assume that one of the following two cases occurs:

$$
\begin{aligned}
& y_{1} \neq x_{2}, \quad y_{2} \neq x_{1} ; \\
& y_{1} \neq x_{2}, \quad y_{2} \neq x_{3}, \quad y_{3} \neq x_{1} .
\end{aligned}
$$

Suppose first that (5.16) holds and choose disjoint neighbourhoods $U_{1}, U_{2}$ of $y_{1}, y_{2}$, respectively, so that

$$
\bar{U}_{1} \subset \overline{\mathbb{R}}^{n} \backslash\left\{x_{2}\right\}, \quad \bar{U}_{2} \subset \overline{\mathbb{R}}^{n} \backslash\left\{x_{1}\right\} .
$$


Fix $x \in \overline{\mathbb{R}}^{n} \backslash\left\{x_{1}, x_{2}\right\}$. Since $U_{1} \cup\{x\}$ is compactly contained in $\overline{\mathbb{R}}^{n} \backslash\left\{x_{2}\right\}$, there exists an integer $j_{2}$ such that

$$
g_{2, j}\left(U_{1} \cup\{x\}\right) \subset U_{2}
$$

for $j>j_{2}$. Similarly, there exists an integer $j_{1}$ such that

$$
g_{1, j}\left(U_{2} \cup\{x\}\right) \subset U_{1}
$$

for $j>j_{1}$. Hence for $j>j_{0}=\max \left(j_{1}, j_{2}\right)$ we have

$$
g_{1, j} \circ g_{2, j}(x) \in U_{1}, \quad g_{2, j} \circ g_{1, j}(x) \in U_{2},
$$

a contradiction since $G$ is abelian and $U_{1}, U_{2}$ are disjoint.

Suppose next that (5.17) holds and choose pairwise disjoint neighbourhoods $U_{1}, U_{2}, U_{3}$ of $y_{1}, y_{2}, y_{3}$, respectively, so that

$$
\bar{U}_{1} \subset \overline{\mathbb{R}}^{n} \backslash\left\{x_{2}\right\}, \quad \bar{U}_{2} \subset \overline{\mathbb{R}}^{n} \backslash\left\{x_{3}\right\}, \quad \bar{U}_{3} \subset \overline{\mathbb{R}}^{n} \backslash\left\{x_{1}\right\} .
$$

If $x \in \overline{\mathbb{R}}^{n} \backslash\left\{x_{1}, x_{2}, x_{3}\right\}$, then as above there exists an integer $j_{0}$ such that for $j>j_{0}$,

$$
g_{2, j}\left(U_{1} \cup\{x\}\right) \subset U_{2}, \quad g_{3, j}\left(U_{2} \cup\{x\}\right) \subset U_{3}, \quad g_{1, j}\left(U_{3} \cup\{x\}\right) \subset U_{1}
$$

from which we obtain

$$
g_{1, j} \circ g_{3, j} \circ g_{2, j}(x) \in U_{1}, \quad g_{2, j} \circ g_{1, j} \circ g_{3, j}(x) \in U_{2},
$$

again contradicting the fact that $G$ is abelian. This completes the proof.

5.18. THEOREM. Each element $g$ in $G$ is elliptic, parabolic, or loxodromic. Moreover, $g$ and $g^{j}$ are always elements of the same type for each integer $j \neq 0$.

Proof. If $g \in G$, then $\langle g\rangle$ is abelian, and hence elementary, and $g$ is elliptic, parabolic, or loxodromic by Theorems 5.7,5.10, and 5.11. Next $g$ is of finite order if and only if $g^{j}$ is. If $g$ is of infinite order, then Theorems 5.10 and 5.11 imply that each element of $\langle g\rangle$ other than the identity is parabolic or loxodromic depending on whether $L(\langle g\rangle)$ contains one or two points, respectively.

We conclude this section with the following consequences of the results proved above. The first is reminiscent of the fact that analytic functions which agree in an open set must agree everywhere.

5.19. Corollary. If $f$ and $g$ in $G$ agree on an open set, then they are identical.

Proof. Let $h=f^{-1} \circ g$. Then $h(x)=x$ in an open set, $h$ is elliptic since card fix $(h)>2$, and hence $h$ is equal to the identity by a classical result of Newman's [25] on periodic homeomorphisms of $\overline{\mathbb{R}}^{n}$.

5.20. Corollary. Suppose that $f$ is a quasiconformal self mapping of $\overline{\mathbb{R}}^{n}$ which has no fixed points and suppose that the iterates of $f$ have uniformly bounded dilatation. Then either $f^{j}$ is equal to the identity for some integer $j$ or there exists a sequence of integers $\left\{j_{k}\right\}$ such that $\left\{f^{j}\right\}$ converges to the identity uniformly in $\overline{\mathbb{B}}^{n}$.

Proof. By hypothesis $F=\langle f\rangle$ is a quasiconformal, and hence convergence, 
group. If the second conclusion fails, then $F$ is discrete and $f$ must be elliptic by Theorem 5.18, since $f$ has no fixed points. Hence the first conclusion holds.

\section{The non-elementary discrete convergence groups}

We consider first how the iterates or powers of an element of a discrete convergence group behave and then apply this information to study the non-elementary groups. As earlier, we continue to let $G$ denote a discrete convergence group acting on $\overline{\mathbb{B}}^{n}$.

Since elliptic elements are of finite order, the convergence properties of $G$ reveal little about the behaviour of the iterates of such an element. The situation is different for elements of infinite order and we have the following result for parabolic elements.

6.1. THEOREM. If $g$ is a parabolic element in $G$ with fixed point $x_{0}$, then

$$
\lim _{j \rightarrow \infty} g^{j}=x_{0} \quad \text { and } \quad \lim _{j \rightarrow \infty} g^{-j}=x_{0}
$$

c-uniformly in $\overline{\mathbb{R}}^{n} \backslash\left\{x_{0}\right\}$.

Proof. The group $\langle g\rangle$ contains only parabolic elements by Theorem 5.18. Hence if $U$ is any neighbourhood of $x_{0}$, Lemma 5.5 with $E_{1}=\left\{x_{0}\right\}$ and $E_{2}=\overline{\mathbb{R}}^{n} \backslash U$ implies that there exists an integer $k$ such that

$$
g^{j}\left(\overline{\mathbb{R}}^{n} \backslash U\right) \subset U \text { and } g^{-j}\left(\overline{\mathbb{R}}^{n} \backslash U\right) \subset U
$$

for $j \geqslant k$.

We establish next the important analogue of Theorem 6.1 for loxodromic elements.

6.2. Theorem. If $g$ is a loxodromic element of $G$ with fixed points $x_{0}, y_{0}$, then these points can be labelled so that

$$
\lim _{j \rightarrow \infty} g^{j}=y_{0} \text { and } \lim _{j \rightarrow \infty} g^{-j}=x_{0}
$$

c-uniformly in $\overline{\mathbb{R}}^{n} \backslash\left\{x_{0}\right\}$ and $\overline{\mathbb{R}}^{n} \backslash\left\{y_{0}\right\}$, respectively. We call $y_{0}$ the attractive and $x_{0}$ the repulsive fixed point for $g$.

Proof. Since $L(\langle g\rangle)=\left\{x_{0}, y_{0}\right\}$, by Lemma 4.1 there exist a sequence $\left\{g_{j}\right\}$ in $\langle g\rangle$ and a point $x_{1} \in L(G)$ such that $g_{j} \rightarrow y_{0}$ and $g_{j}^{-1} \rightarrow x_{1} c$-uniformly in $\overline{\mathbb{R}}^{n} \backslash\left\{x_{1}\right\}$ and $\overline{\mathbb{R}}^{n} \backslash\left\{y_{0}\right\}$, respectively; since $x_{0}$ is fixed by each $g_{j}$, it follows that $x_{1}=x_{0}$. Hence by passing to a subsequence and relabelling the fixed points, if necessary, we obtain integers $k(j)>0$ such that

$$
\lim _{j \rightarrow \infty} g^{k(j)}=y_{0} \quad \text { and } \quad \lim _{j \rightarrow \infty} g^{-k(j)}=x_{0}
$$

$c$-uniformly in $\mathbb{\mathbb { R }}^{n} \backslash\left\{x_{0}\right\}$ and $\overline{\mathbb{R}}^{n} \backslash\left\{y_{0}\right\}$, respectively.

Let $U, V$ be disjoint chordal balls about $x_{0}, y_{0}$, respectively. We shall show that 
there exists an integer $k>0$ such that

$$
g^{j}\left(\overline{\mathbb{R}}^{n} \backslash U\right) \subset V \quad \text { and } \quad g^{-j}\left(\overline{\mathbb{R}}^{n} \backslash V\right) \subset U
$$

for $j \geqslant k$. Since $g$ fixes $y_{0}$, we may assume that $g(V) \cap U=\varnothing$.

Let $E=\overline{\mathbb{R}}^{n} \backslash(U \cup V)$. Then Corollary 3.10 with $E_{1}=\left\{x_{0}\right\}, E_{2}=\left\{y_{0}\right\}$, and $E_{3}=E$ implies that

$$
g^{j}(E) \cap E=\varnothing \quad \text { and } \quad g^{-j}(E) \cap E=\varnothing
$$

for all but finitely many $j>0$. Hence with (6.3) we can choose an integer $k>0$ such that (6.4) holds for $j=k$ and (6.5) holds for $j \geq k$.

If (6.4) holds for some $j=i \geqslant k$, then

$$
g^{i+1}(E) \cap U \subset g\left(g^{i}\left(\overline{\mathbb{R}}^{n} \backslash U\right)\right) \cap U \subset g(V) \cap U=\varnothing,
$$

so that $g^{i+1}(E) \subset V$ by $(6.5)$. Then

$$
g^{i+1}(\partial V) \subset g^{i+1}(E) \subset V
$$

whence $g^{i+1}(V) \subset V$ and we obtain the first part of (6.4) for $j=i+1$. The second part follows similarly for $j=i+1$ and hence (6.4) holds for all $j \geqslant k$ by induction.

6.6. Corollary. Suppose that $g$ is an element of $G$. Then $g$ is loxodromic if and only if there exist an integer $j$ and a topological ball $U$ such that $g^{j}(\bar{U}) \subset U$.

Proof. By Lemma 5.3, $h=g^{j}$ is loxodromic if $g^{j}(\bar{U}) \subset U$ for some integer $j$ and topological ball $U$; hence $g$ is loxodromic by Theorem 5.18. Conversely, if $g$ is loxodromic and if $U$ is any neighbourhood of one of the fixed points whose closure does not contain the other, then $g^{j}(\bar{U}) \subset U$ for some integer $j$ by Theorem 6.2 .

Thus the criterion in Lemma 5.3 essentially characterizes loxodromic elements in a discrete convergence group. We show next that the fixed point sets for pairs of loxodromic elements either are disjoint or coincide.

6.7. THEOREM. If $f$ and $g$ in $G$ have a fixed point in common and if $g$ is loxodromic, then $f$ and $g$ have two fixed points in common and there exists a non-zero integer $k$ such that $f \circ g^{k}=g^{k} \circ f$.

Proof. Suppose that $x_{0}$ is a fixed point of $f$ and that $x_{0}$ and $y_{0}$ are respectively the repulsive and attractive fixed points for $g$. Next let $U, V$ be disjoint chordal balls about $x_{0}, y_{0}$, respectively, chosen so that $g(U) \cap V=\varnothing$, and let $E=\overline{\mathbb{R}}^{n} \backslash(U \cup V)$. By Theorem 6.2, there is an integer $j_{0}$ such that

$$
f \circ g^{-j}(E) \subset U \backslash\left\{x_{0}\right\}
$$

for $j \geqslant j_{0}$. Theorem 6.2 also implies that for each such $j$,

$$
g^{k} \circ f \circ g^{-j}(E) \backslash U \neq \varnothing
$$

for sufficiently large $k$. Let $k(j)$ denote the smallest $k$ for which (6.8) holds and set $h_{j}=g^{k(j)} \circ f \circ g^{-j}$. Then

$$
h_{j}\left(x_{0}\right)=x_{0}, \quad h_{j}(E) \cap E \neq \varnothing,
$$


for $j>j_{0}$, while

$$
\lim _{j \rightarrow \infty} h_{j}\left(y_{0}\right)=y_{0}
$$

since $k(j) \rightarrow \infty$ as $j \rightarrow \infty$. Hence for large $j$, the closed sets

$$
E_{1}=\left\{x_{0}\right\}, \quad E_{2}=\left\{h_{k}\left(y_{0}\right): k \geqslant j\right\} \cup\left\{y_{0}\right\}, \quad E_{3}=E
$$

are pairwise disjoint and Corollary 3.10 implies that there exist $j_{1}, j_{2}$ with $j_{1}<j_{2}$ for which $h_{j_{1}}=h_{j_{2}}$ whence

$$
g^{k}=f \circ g^{j} \circ f^{-1},
$$

where $j=j_{2}-j_{1}$ and $k=k\left(j_{2}\right)-k\left(j_{1}\right)$. From this it follows that

$$
g^{k m}=f \circ g^{j m} \circ f^{-1}
$$

for all integers $m$, and that $f\left(y_{0}\right)=y_{0}$.

We want to show that $k=j$. If $k<j$, we can choose $m>0$ such that

$$
g^{(k-j) m}(f(\bar{U})) \subset U \text { and } g^{(j-k) m}(f(\bar{V})) \subset V .
$$

Then $U_{0}=g^{i m}(U)$ is a topological ball, $\bar{U}_{0}$ does not contain $y_{0}$, and

$$
f\left(\bar{U}_{0}\right)=f\left(g^{j m}(\bar{U})\right)=g^{k m}(f(\tilde{U})) \subset g^{k m}\left(g^{(j-k) m}(U)\right)=U_{0} .
$$

Hence $f$ is loxodromic by Lemma 5.3 and since $f$ fixes $x_{0}, f$ has $x_{0}$ as its attractive fixed point. Similarly $V_{0}=g^{-j m}(V)$ does not contain $x_{0}$ in its closure, while

$$
f\left(\bar{V}_{0}\right)=f\left(g^{-j m}(\bar{V})\right)=g^{-k m}(f(\bar{V})) \subset g^{-k m}\left(g^{(k-j) m}(V)\right)=V_{0} .
$$

Thus $x_{0}$ cannot be the attractive fixed point for $f$ and we conclude that $k \geqslant j$. Reversing the roles of $f$ and $f^{-1}$ in the above argument shows that $k \leqslant j$ and hence that $k=j$. Thus $f \circ g^{k}=g^{k} \circ f$.

\subsection{Corollary. If $f, g$ in $G$ are loxodromic, then either}

$$
\operatorname{fix}(f) \cap \operatorname{fix}(g)=\varnothing \quad \text { or } \operatorname{fix}(f)=\operatorname{fix}(g) .
$$

In the latter case there exist non-zero integers $j$ and $k$ such that $f^{j}=g^{k}$.

Proof. Theorem 6.7 implies that $\operatorname{fix}(f)$ and $\operatorname{fix}(g)$ are disjoint or coincide. Hence it remains only to establish the existence of the integers $j$ and $k$ in the second case. For this let $x_{0}, y_{0}$ and $U, V, E$ be as in the proof of Theorem 6.7. By replacing $f$ by $f^{-1}$ we may further assume that $x_{0}$ and $y_{0}$ are, respectively, the repulsive and attractive fixed points for $f$ as well as $g$. Then

$$
f^{-j}(E) \subset U \backslash\left\{x_{0}\right\},
$$

for $j \geqslant j_{0}$, and as in the proof of Theorem 6.7 we can find for each such $j$ an integer $k(j)$ such that $h_{j}(E) \cap E \neq \varnothing$, where $h_{j}=g^{k(j)} \circ f^{-j}$. Corollary 3.10 with $E_{1}=\left\{x_{0}\right\}, E_{2}=\left\{y_{0}\right\}$, and $E_{3}=E$ then implies that $h_{j_{1}}=h_{j_{2}}$ for some $j_{1}<j_{2}$ and hence that $f^{j}=g^{k}$ where $j=j_{2}-j_{1}$ and $k=k\left(j_{2}\right)-k\left(j_{1}\right)$.

We now apply Theorem 6.7 to show that a non-elementary group contains infinitely many loxodromic elements and that its limit set is the closure of the set of fixed points of these elements. 
6.10. THEOREM. If $G$ is non-elementary, then $\operatorname{fix}(G)=\varnothing$ and $L(x, G)=L(G)$ for each point $x$ in $\overline{\mathbb{R}}^{n}$.

Proof. Lemma 5.8 implies that $G$ contains at least one loxodromic element $f$. To establish the first conclusion we show there exists a second element $g \in G$ such that

$$
\operatorname{fix}(f) \cap \operatorname{fix}(g)=\varnothing .
$$

Choose $y_{1} \in L(G) \backslash f i x(f)$. By Lemma 4.1 there exist a point $x_{1} \in L(G)$ and a sequence $\left\{g_{j}\right\}$ in $G$ which converges to $y_{1} c$-uniformly in $\mathbb{R}^{n} \backslash\left\{x_{1}\right\}$. Suppose that

$$
\begin{gathered}
\operatorname{fix}(f) \cap \operatorname{fix}\left(g_{j}\right) \neq \varnothing, \\
\operatorname{fix}(f) \cap g_{j}(\operatorname{fix}(f)) \neq \varnothing
\end{gathered}
$$

hold for all $j$. Then $x_{1} \in \mathrm{fix}(f)$, since otherwise $g_{j}$ would converge to $y_{1}$ uniformly in $\operatorname{fx}(f)$ and (6.12) would not hold for large $j$. Hence $x_{1} \neq y_{1}$, all but a finite number of the $g_{j}$ are loxodromic by Lemma 5.4, and $\operatorname{fix}(f)=\operatorname{fix}\left(g_{j}\right)$ for large $j$ by (6.11) and Corollary 6.9. If $f(x)=\left\{x_{0}, y_{0}\right\}$ and $x_{1}=x_{0}$, then

$$
y_{0}=\lim _{j \rightarrow \infty} g_{j}\left(y_{0}\right)=y_{1}
$$

and we have a contradiction. Hence (6.11) or (6.12) fails for some $j$ and we may take $g=g_{j}$ or $g=g_{j} \circ f \circ g_{j}^{-1}$, respectively.

The second conclusion then follows directly from Lemma 4.4.

6.13. Theorem. If $G$ is non-elementary, then $L(G)$ is the smallest closed non-empty $G$-invariant subset of $\overline{\mathbb{R}}^{n}$.

Proof. If $x$ is a point of a closed $G$-invariant set $E$, then $L(G)=L(x, G) \subset E$ by Theorem 6.10 ; Lemma 2.4 implies that $L(G)$ is such a set.

6.14. Remark. Theorem 6.13 yields a great deal of information about the limit set $L(G)$ of a non-elementary group. For example, it implies that $L(G)$ lies in the closure of any non-empty $G$-invariant set in $\overline{\mathbb{R}}^{n}$.

6.15. Corollary. If $G$ is non-elementary, then $G$ contains infinitely many loxodromic elements, no two of which have a common fixed point, and $L(G)$ is the closure of the set of fixed points of the loxodromic elements in $G$.

6.16. Corollary. If $G$ is non-elementary and if $G$ contains an element $g$ which is either parabolic or is elliptic with $\operatorname{fix}(g) \neq \varnothing$, then $L(G)$ lies in the closure of the set of fixed points of the parabolic or elliptic elements in $G$, respectively.

Proofs. Let $F_{E}, F_{P}, F_{L}$ denote the set of fixed points for the elliptic, parabolic, loxodromic elements in $G$. If $f$ and $g$ are in $G$, then $g \circ f \circ g^{-1}$ is the same type of element as $f$ with $\operatorname{fix}\left(g \circ f \circ g^{-1}\right)=g(\operatorname{fix}(f))$. Hence each of the above sets is $G$-invariant.

Lemma 5.8 implies that $F_{L}$ is non-empty; $F_{E}$ and $F_{P}$ are non-empty by hypothesis. The conclusions for Corollaries 6.15 and 6.16 now follow from Corollary 6.9 and Theorem 6.13 . 
We establish next an extension of Corollary 6.15 which says that the fixed point pairs $\left(x_{0}, y_{0}\right)$ of loxodromic elements are dense in $L(G) \times L(G)$.

6.17. THEOREM. If $G$ is non-elementary and if $V_{1}$ and $V_{2}$ are disjoint open sets both of which intersect $L(G)$, then there exists a loxodromic element $g$ in $G$ with one fixed point in $V_{1}$ and the other in $V_{2}$.

Proof. We may clearly assume that $V_{1}$ and $V_{2}$ are chordal balls which both meet $L(G)$. Then by Corollary 6.15 , for $k=1,2$ there exists a loxodromic element $g_{k} \in G$ with fixed points $x_{k}, y_{k}$ and $y_{k} \in V_{k}$. By replacing $g_{k}$ by $g_{k}^{-1}$, if necessary, we may assume that $y_{k}$ is the attractive fixed point for $g_{k}$. Since $V_{1}$ and $V_{2}$ are disjoint, $y_{1} \neq y_{2}$ and hence $x_{1} \neq x_{2}$ by Corollary 6.9. Again by Corollary 6.15 there exists a loxodromic element $g_{0}$ with $x_{0}$ and $y_{0}$ as its repulsive and attractive fixed points such that

$$
\left\{x_{0}, y_{0}\right\} \cap\left\{x_{1}, x_{2}\right\}=\varnothing \text {. }
$$

Let $U_{0}, V_{0}, U_{1}, U_{2}$ be pairwise disjoint neighbourhoods of $x_{0}, y_{0}, x_{1}, x_{2}$, respectively. Then

$$
\bar{V}_{2} \subset \overline{\mathbb{R}}^{n} \backslash\left\{y_{1}\right\}, \quad \bar{U}_{1} \subset \overline{\mathbb{R}}^{n} \backslash\left\{x_{0}\right\}, \quad \bar{V}_{0} \subset \overline{\mathbb{R}}^{n} \backslash\left\{x_{2}\right\},
$$

and we obtain

$$
g_{1}^{-j}\left(\bar{V}_{2}\right) \subset U_{1}, \quad g_{0}^{j}\left(U_{1}\right) \subset V_{0}, \quad g_{2}^{j}\left(V_{0}\right) \subset V_{2},
$$

for large $j$. Similarly,

$$
g_{2}^{-j}\left(\bar{V}_{1}\right) \subset U_{2}, \quad g_{0}^{-j}\left(U_{2}\right) \subset U_{0}, \quad g_{1}^{j}\left(U_{0}\right) \subset V_{1},
$$

for large $j$. Hence we can choose $j$ such that

$$
h_{j}\left(\bar{V}_{2}\right) \subset V_{2}, \quad h_{j}^{-1}\left(\bar{V}_{1}\right) \subset V_{1},
$$

where $h_{j}=g_{2}^{j} \circ g_{0}^{j} \circ g_{1}^{-j}$. Then by Lemma 5.3, $g=h_{j}$ is a loxodromic element which has fixed points in $V_{1}$ and $V_{2}$.

We conclude this section with some topological properties of the limit set of a non-elementary convergence group.

6.18. LEMMA. If $g$ is a loxodromic element in $G$, then the fixed points of $g$ either are point components of $L(G)$ or they lie in the same non-degenerate component of $L(G)$.

Proof. Suppose the repulsive and attractive fixed points $x_{0}, y_{0}$ belong to different components $C\left(x_{0}\right), C\left(y_{0}\right)$ of $L(G)$. Since these components are invariant under $g$ and since $g^{j} \rightarrow y_{0}$ and $g^{-j} \rightarrow x_{0}$ uniformly in $C\left(y_{0}\right)$ and $C\left(x_{0}\right)$, respectively,

$$
\begin{aligned}
& q\left(C\left(y_{0}\right)\right)=\lim _{j \rightarrow \infty} q\left(g^{j}\left(C\left(y_{0}\right)\right)\right)=0, \\
& q\left(C\left(x_{0}\right)\right)=\lim _{j \rightarrow \infty} q\left(g^{-j}\left(C\left(x_{0}\right)\right)\right)=0,
\end{aligned}
$$

where $q(C)$ denotes the chordal diameter of $C$. 
6.19. THEOREM. If $G$ is non-elementary and if $C$ is a component of $L(G)$, then either $C=L(G)$ or $C$ lies in the closure of $L(G) \backslash C$.

Proof. Choose $x_{0} \in C$, suppose there exists a point $y_{0} \in L(G) \backslash C$, and let $U, V$ denote disjoint neighbourhoods of $x_{0}, y_{0}$ such that $V \cap C=\varnothing$. By Theorem 6.17 there exists a loxodromic element $g \in G$ with fixed points $x_{1} \in U$ and $y_{1} \in V$. If $C$ is non-degenerate, then $x_{1}$ cannot lie in $C$ since otherwise by Lemma $6.18, y_{1}$ would also lie in $C$ and hence in $V \cap C$; thus

$$
x_{1} \in(L(G) \backslash C) \cap U .
$$

If $C=\left\{x_{0}\right\}$, then because $L(G)$ is perfect there exists a point $x_{1}$ for which $(6.20)$ holds.

6.21. Corollary. If $G$ is non-elementary and if $L(G)$ is not connected, then $L(G)$ is not locally connected at any point of $L(G)$.

Proof. Let $U$ be a neighbourhood of a point $x_{0} \in L(G)$ and let $C$ denote the component of $L(G)$ containing $x_{0}$. By Theorem 6.19 there exists a point $x_{1} \in(L(G) \backslash C) \cap U$ and hence $L(G)$ is not locally connected at $x_{0}$.

We say that a point $x$ of an arcwise connected continuum $C$ is an endpoint if there is no arc in $C$ which contains $x$ as an interior point.

6.22. THEOREM. Suppose that $G$ is non-elementary and that $L(G)$ is locally connected. Then $L(G)$ is arcwise connected, and either $L(G)$ contains a simple closed curve or the fixed points of every loxodromic element in $G$ are endpoints of $L(G)$.

Proof. Corollary 6.21 implies that $L(G)$ is connected and hence arcwise connected [12]. Suppose that there exists a loxodromic element $g \in G$ with fixed points $x_{0}, y_{0}$ where $x_{0}$ is not an endpoint of $L(G)$; by replacing $g$ by $g^{-1}$ we may assume that $y_{0}$ is the attractive fixed point for $g$. Then there exists an arc $\alpha \subset L(G)$ with endpoints $x_{1}, x_{2}$ which contains $x_{0}$ as an interior point. Let $U$ be a neighbourhood of $y_{0}$ which does not contain $x_{0}$. Because $L(G)$ is locally connected there exists a neighbourhood $V$ of $y_{0}$ such that each pair of points in $V \cap L(G)$ can be joined by an arc in $U \cap L(G)$ [12, pp. 113-118]. By Theorem 6.2 we can choose an integer $j$ such that $g^{j}\left(x_{1}\right)$ and $g^{j}\left(x_{2}\right)$ lie in $V \cap L(G)$ and hence can be joined by an arc $\beta$ in $U \cap L(G)$. Since $x_{0} \in g^{j}(\alpha) \backslash \beta, g^{j}(\alpha) \cup \beta$ contains a simple closed curve which lies in $L(G)$.

\section{Groups of homeomorphisms}

We saw in $\$ 6$ that the iterates of elements of a discrete convergence group behave in a very regular fashion. We consider now what conditions on an arbitrary group $G$ of self homeomorphisms of $\overline{\mathbb{R}}^{n}$ allow one to conclude that $G$ is a discrete convergence group. A sufficient condition is given in Theorem 7.8.

Next, the fact that we can classify the elements $g$ of a discrete convergence group $G$ in exactly the same way as elements of a Möbius group makes it natural to ask if each such $g$ is the topological conjugate of a Möbius transformation $h$. 
More important, is each convergence group $G$ topologically conjugate to a Möbius group $H$ ? We provide examples to show that, except in the case of a loxodromic element, the answer to both questions is, in general, 'no'.

We assume throughout this section that $g$ is an arbitrary self homeomorphism of $\bar{R}^{n}$ and that $G$ is a group of such elements. Theorems 6.1 and 6.2 suggest how to extend the classification of Definition 5.1 to elements $g$ of $G$.

7.1. Definition. We say that $g$ is elliptic if $\operatorname{ord}(g)<\infty$, that $g$ is parabolic if there exists a point $x_{0}$ such that

$$
\lim _{j \rightarrow \infty} g^{j}=x_{0} \quad \text { and } \quad \lim _{j \rightarrow \infty} g^{-j}=x_{0}
$$

$c$-uniformly in $\overline{\mathbb{R}}^{n} \backslash\left\{x_{0}\right\}$, and that $g$ is loxodromic if there exist two points $x_{0}, y_{0}$ such that

$$
\lim _{j \rightarrow \infty} g^{j}=y_{0} \quad \text { and } \quad \lim _{j \rightarrow \infty} g^{-j}=x_{0}
$$

$c$-uniformly in $\overline{\mathbb{R}}^{n} \backslash\left\{x_{0}\right\}$ and $\overline{\mathbb{R}}^{n} \backslash\left\{y_{0}\right\}$, respectively.

7.4. Lemma. The homeomorphism $g$ is elliptic, parabolic, or loxodromic according to Definition 7.1 if and only if $\langle g\rangle$ is a discrete convergence group and $g$ is an elliptic, parabolic, or loxodromic element of $\langle g\rangle$, respectively.

Proof. We may exclude the elliptic case where there is nothing to prove. Then the necessity follows from Theorems 6.1 and 6.2. For the sufficiency, (7.2) or (7.3) imply that ord $(g)=\infty$ and that $\langle g\rangle$ is a discrete convergence group with $L(\langle g\rangle)=\left\{x_{0}\right\}$ or $L(\langle g\rangle)=\left\{x_{0}, y_{0}\right\}$, respectively. Then $g$ is a parabolic or loxodromic element of $\langle g\rangle$ with $\operatorname{fix}(g)=\left\{x_{0}\right\}$ or $\operatorname{fix}(g)=\left\{x_{0}, y_{0}\right\}$ by Theorems 5.10 and 5.11, respectively.

The classification in Definition 7.1 is clearly not exhaustive for the elements of an arbitrary group $G$. Moreover, Example 7.20 shows that $G$ need not be a convergence group even if it contains only elements of the three types described above. However, we prove next that $G$ is a convergence group if it is properly discontinuous in $\overline{\mathbb{P}} \backslash E$ where $E$ is closed and has only point components. We begin with the following result.

7.5. Lemma. Suppose that $E$ is a totally disconnected closed set, that $\left\{r_{k}\right\}$ is a sequence of positive numbers which converge to 0 and that $\left\{D_{k}\right\}$ is a nondecreasing sequence of domains in $\overline{\mathbb{R}}^{n} \backslash E$ such that

$$
\sup _{x \in \partial D_{k}} q(x, E) \leqslant r_{k}
$$

for each $k$. If $x_{0}$ is in $E$ and if $C_{k}$ is the component of $\overline{\mathbb{R}}^{n} \backslash D_{k}$ which contains $x_{0}$, then

$$
\lim _{k \rightarrow \infty} q\left(C_{k}\right)=0
$$

Proof. Suppose that (7.7) does not hold. Then $\left\{C_{k}\right\}$ is a non-increasing sequence of continua whose intersection is a continuum $C$ which contains $x_{0}$ [32]. 
Since $E$ is totally disconnected, there exists a point $y_{0} \in C \backslash E$ which can be joined by an arc $\alpha \subset \mathbb{R}^{n} \backslash E$ to a point $z_{0} \in D_{1}$. Fix $k$ so that $r_{k}<q(\alpha, E)$. Then $z_{0} \in \alpha \cap D_{k}$ while $\alpha \cap \partial D_{k}=\varnothing$ by (7.6). Hence $\alpha \subset D_{k}$ which contradicts the fact that $y_{0} \in C \subset \overline{\mathbb{R}}^{n} \backslash D_{k}$.

7.8. THEOREM. If $E$ is a totally disconnected closed set and if $G$ is properly discontinuous in $\mathbb{R}^{n} \backslash E$, then $G$ is a discrete convergence group with $L(G) \subset E$.

Proof. By (2.3), $L(G) \subset E$ and hence $G$ is discontinuous and discrete. Thus it remains to show that for each infinite sequence of elements in $G$ there exist a subsequence $\left\{g_{j}\right\}$ and points $x_{0}, y_{0}$ such that $g_{j} \rightarrow y_{0}$ and $g_{j}^{-1} \rightarrow x_{0} c$-uniformly in $\mathbb{R}^{n} \backslash\left\{x_{0}\right\}$ and $\mathbb{R}^{n} \backslash\left\{y_{0}\right\}$ respectively; here $x_{0}$ and $y_{0}$ need not be distinct.

Fix $z_{0} \in \overline{\mathbb{R}}^{n} \backslash E$. By compactness we can choose a subsequence $\left\{g_{j}\right\}$ of the given sequence and points $x_{0}, y_{0}$ such that

$$
\lim _{j \rightarrow \infty} g_{j}\left(z_{0}\right)=y_{0}, \quad \lim _{j \rightarrow \infty} g_{j}^{-1}\left(z_{0}\right)=x_{0}
$$

Then $x_{0}, y_{0} \in L(G) \subset E$ as in the proof of Lemma 4.4. Next let $U, V$ be neighbourhoods of $x_{0}, y_{0}$, respectively. We shall complete the proof by showing there exists a $j_{0}$ such that

$$
g_{j}\left(\overline{\mathbb{R}}^{n} \backslash U\right) \subset V, \quad g_{j}^{-1}\left(\overline{\mathbb{R}}^{n} \backslash V\right) \subset U,
$$

whenever $j \geqslant j_{0}$.

Let $\left\{r_{k}\right\}$ be a decreasing sequence of numbers in $\left(0, q\left(z_{0}, E\right)\right)$ which converge to 0 , and set

$$
E_{k}=\left\{x \in \overline{\mathbb{R}}^{n}: q(x, E) \leqslant r_{k}\right\}
$$

for $k=1,2, \ldots$. Next let $D_{k}$ denote the component of $\mathbb{R}^{n} \backslash E_{k}$ which contains $z_{0}$. Then $\partial D_{k} \subset E_{k}$, the domains $D_{k}$ satisfy the hypotheses of Lemma 7.5, and hence for each $x \in E$,

$$
\lim _{k \rightarrow \infty} q\left(C_{k}(x)\right)=0 \text {, }
$$

where $C_{k}(x)$ denotes the component of $\overline{\mathbb{R}}^{n} \backslash D_{k}$ which contains $x$. Thus we can fix $k=k_{0}$ such that

$$
C\left(x_{0}\right)=C_{k}\left(x_{0}\right) \subset U, \quad C\left(y_{0}\right)=C_{k}\left(y_{0}\right) \subset V,
$$

and such that $C\left(x_{0}\right) \neq C\left(y_{0}\right)$ if $x_{0} \neq y_{0}$. Then since $D=D_{k}$ is compactly contained in $\overline{\mathbb{R}}^{n} \backslash E$, there exists a $j_{0}$ such that

$$
g_{j}(D) \cap D=\varnothing, \quad g_{j}^{-1}(D) \cap D=\varnothing,
$$

and, with (7.9), such that

$$
y_{j}=g_{j}\left(z_{0}\right) \in C\left(y_{0}\right), \quad x_{j}=g_{j}^{-1}\left(z_{0}\right) \in C\left(x_{0}\right)
$$

whenever $j \geqslant j_{0}$.

Suppose that $j \geqslant j_{0}$. Since $D$ is connected, (7.12) implies that $g_{j}(D)$ and $g_{j}^{-1}(D)$ each lie in a component of $\overline{\mathbb{R}}^{n} \backslash D$. If $C_{1}, C_{2}$ are different components of $\mathbb{R}^{n} \backslash D$ and if

$$
g_{j}(D) \subset C_{1}, \quad g_{j}\left(C_{2}\right) \backslash C_{1} \neq \varnothing,
$$


then $\overline{\mathbb{R}}^{n} \backslash C_{1}$ is connected [32, Theorem I.9.11] while

$$
\begin{aligned}
& \partial g_{j}\left(C_{2}\right) \cap\left(\overline{\mathbb{R}}^{n} \backslash C_{1}\right) \subset g_{j}(\bar{D}) \cap\left(\overline{\mathbb{R}}^{n} \backslash C_{1}\right)=\varnothing, \\
& g_{j}\left(C_{2}\right) \cap\left(\overline{\mathbb{R}}^{n} \backslash C_{1}\right) \neq \varnothing .
\end{aligned}
$$

Thus $\overline{\mathbb{B}}^{n} \backslash C_{1} \subset g_{j}\left(C_{2}\right)$, whence

$$
\begin{aligned}
& g_{j}\left(\overline{\mathbb{R}}^{n} \backslash C_{2}\right) \subset C_{1}, \\
& g_{j}^{-1}(D) \subset C_{2} .
\end{aligned}
$$

Since $z_{0} \in D,(7.13)$ implies that

$$
y_{j} \in C\left(y_{0}\right) \cap g_{j}(D), \quad x_{j} \in C\left(x_{0}\right) \cap g_{j}^{-1}(D),
$$

and hence that

$$
g_{j}(D) \subset C\left(y_{0}\right), \quad g_{j}^{-1}(D) \subset C\left(x_{0}\right) .
$$

If $x_{0} \neq y_{0}$, then $C\left(x_{0}\right), C\left(y_{0}\right)$ are different components of $\overline{\mathbb{R}}^{n} \backslash D$,

$$
z_{0} \in D \subset g_{j}\left(C\left(x_{0}\right)\right) \backslash C\left(y_{0}\right)
$$

by (7.18), and (7.14) holds with $C_{1}=C\left(y_{0}\right)$ and $C_{2}=C\left(x_{0}\right)$. Thus

$$
g_{j}\left(\overline{\mathbb{R}}^{n} \backslash C\left(x_{0}\right)\right) \subset C\left(y_{0}\right)
$$

by (7.15). This and (7.11) imply the first half of (7.10). The second half follows similarly.

Finally, if $x_{0}=y_{0}$, then $C\left(x_{0}\right)=C\left(y_{0}\right)$ and

$$
g_{j}\left(\overline{\mathbb{R}}^{n} \backslash C\left(y_{0}\right)\right) \subset C\left(y_{0}\right) .
$$

For otherwise we could find a component $C$ of $\mathbb{R}^{n} \backslash D$ different from $C\left(y_{0}\right)$ such that $g_{j}(C) \backslash C\left(y_{0}\right) \neq \varnothing,(7.14)$ would hold with $C_{1}=C\left(y_{0}\right)$ and $C_{2}=C$, and we would obtain

$$
g_{j}^{-1}(D) \subset C\left(x_{0}\right) \cap C=C\left(y_{0}\right) \cap C
$$

from (7.16) and (7.18), a contradiction. The first half of (7.10) is now a consequence of (7.11) and (7.19); the proof for the second half is similar.

The proof of Theorem 7.8 depends crucially on (7.7) of Lemma 7.5. There the hypothesis on $E$ was used to conclude that $E$ contains no continua and that $\overline{\mathbb{R}}^{n} \backslash E$ is connected. The following example shows that this second property is not sufficient to imply the desired conclusion in Theorem 7.8 .

7.20. ExAmple. For each tamely imbedded Jordan curve $C$ in $\overline{\mathbb{R}}^{n}$ there exists a group $G$ with the following properties: $G$ contains only parabolic elements besides the identity, $G$ is properly discontinuous in $\overline{\mathbb{R}}^{n} \backslash C$ with $L(G)=C$, and $G$ is not a convergence group.

Proof. Let $a$ be any irrational number and let $G$ be the group generated by

$$
\begin{aligned}
& g_{1}\left(x_{1}, x_{2}, \ldots, x_{n}\right)=\left(x_{1}+a, x_{2}, \ldots, x_{n}\right), \\
& g_{2}\left(x_{1}, x_{2}, \ldots, x_{n}\right)=\left(x_{1}+1,2 x_{2}, \ldots, 2 x_{n}\right) .
\end{aligned}
$$


Then each $g \in G$ is of the form

$$
g\left(x_{1}, x_{2}, \ldots, x_{n}\right)=\left(x_{1}+p a+q, 2^{q} x_{2}, \ldots, 2^{q} x_{n}\right),
$$

where $\bar{p}, q=0, \pm 1, \pm 2, \ldots$. If $g$ is not the identity, then $p a+q \neq 0$ and $g$ satisfies (7.2) with $x_{0}=\infty$.

Let $L$ denote the closure of the $x_{1}$-axis. From the above representation we see that each sequence in $G$ contains a subsequence $\left\{g_{j}\right\}$ such that

$$
\lim _{j \rightarrow \infty} g_{j}=\infty \text { or } \lim _{j \rightarrow \infty} g_{j}^{-1}=\infty
$$

$c$-uniformly in $\overline{\mathbb{R}}^{n} \backslash L$; hence $G$ is properly discontinuous in $\overrightarrow{\mathbb{R}}^{n} \backslash L$. Next for each integer $t>1$, there exist integers $p$ and $q$ such that $1 \leqslant p \leqslant t$ and $|p a+q|<1 / t$ [20, Theorem 7.1]. This implies the existence of a sequence $\left\{g_{j}^{*}\right\}$ in $G$ such that

$$
\lim _{j \rightarrow \infty} g_{j}^{*}(x)=x \text { and } \quad \lim _{j \rightarrow \infty} g_{j}^{*-1}(x)=x
$$

for all $x \in L$. Hence $L(G)=L$ while (7.21), with $g_{j}=g_{j}^{*}$, and (7.22) show that $G$ does not have the convergence property. Thus $G$ is the desired group for the case where $C=L$.

For the general case let $f$ be a self homeomorphism of $\overline{\mathbb{R}}^{n}$ for which $f(L)=C$ and replace $G$ by $f \circ G \circ f^{-1}$.

7.23. Remarks. It is easy to modify the above construction and obtain a group $G$ such that $G$ contains, besides the identity, only loxodromic elements with common fixed points, $G$ is properly discontinuous in $\overline{\mathbb{R}}^{n}$ with $L(G)=C$, and $G$ is not a convergence group.

Many of the results derived for discrete convergence groups in $\$ \$ 4,5$, and 6 do not hold for arbitrary discrete groups $G$. For example, the group $G$ in Example 7.20 is abelian but non-elementary; the group cited above is non-elementary while its loxodromic elements all have the same fixed points.

We turn now to the question of deciding if convergence groups are topologically conjugate to conformal groups.

7.24. Definition. We say that an element $g$ of $G$ is standard if it is topologically conjugate to a Möbius transformation $h$, and that $G$ is standard if it is topologically conjugate to a Möbius group $H$.

Since the classification in Definition 7.1 is invariant with respect to topological conjugation, a homeomorphism which is conjugate to an element of a discrete Möbius group must be elliptic, parabolic, or loxodromic. We consider what can be said about each of these classes.

7.25. Elliptic elements. By a theorem due in part to Brouwer, Kerékjârtó, and Eilenberg [5], each periodic self homeomorphism $f$ of $S^{2}$ is topologically conjugate to an orthogonal transformation.

The situation is different in higher dimensions. Montgomery and Zippin constructed in [23] a self homeomorphism $f$ of $S^{3}$ with period 2 where fix $(f)$ is a 
wild knot; hence $f$ is not topologically conjugate to an orthogonal transformation. More exotic examples can be found among counter-examples to the generalized Smith conjecture. In particular, Giffen [10] has shown that for $n \geqslant 4$ there exist smooth, and hence quasiconformal, periodic orientation-preserving homeomorphisms $f$ of $S^{n}$ which are not conjugate to an orthogonal transformation. The recent affirmative solution of the Smith conjecture shows that no such examples exist when $n=3$ [24]. In summary we have the following result.

7.26. THEOREM. When $n=2$, each elliptic element $g$ is standard. For each $n \geqslant 3$ there exists a non-standard elliptic element $g$ and, when $n \geqslant 4$, we can choose $g$ so that it is quasiconformal.

7.27. COROLlaRy. For each $n \geqslant 3$ there exists a finite non-standard convergence group $G$.

Proof. If $g$ is the element in Theorem 7.26 , then $\langle g\rangle$ is a finite convergence group which is not standard.

7.28. Parabolic elements. If $g$ is parabolic with fixed point $x_{0}=\infty$, then (7.2) says that

$$
\lim _{j \rightarrow \infty} g^{j}=\infty \text { and } \lim _{j \rightarrow \infty} g^{-j}=\infty
$$

$c$-uniformly in $\mathbb{R}^{n}$. This is known as Sperner's condition and the orientationpreserving self homeomorphisms $g$ of $\mathbb{R}^{n}$ which satisfy it are called quasitranslations. Obviously each such mapping has a homeomorphic extension to $\overline{\mathbb{R}}^{n}$.

By results due to Sperner and Kérékjárto [16], each quasitranslation in $\mathbb{R}^{2}$ is a topological translation, that is, it is topologically conjugate to a mapping of the form $f(x)=x+e_{1}$. Again the situation is different in higher dimensions, and for each $n \geqslant 3$, Kinoshita [18] and Husch [14] have exhibited quasitranslations of $\mathbb{R}^{n}$ which are not topological translations.

7.29. LemmA. Each sense-preserving parabolic Möbius transformation $h$ is a topological translation.

Proof. We may assume that $h$ fixes $\infty$. Then by [1, pp. 47-49], $h(x)=u(x)+a$ where $u$ is an orthogonal transformation and $a$ considered as an $n$-vector has a non-zero projection $b$ onto the $k$-plane fix $(u)$, where $1 \leqslant k \leqslant n-2$. Let $E$ denote the $(n-1)$-plane through the origin orthogonal to $b$. If $x_{0} \in E$, then

$$
h\left(x_{0}\right)=u\left(x_{0}\right)+a=x_{1}+b \quad \text { where } x_{1} \in E .
$$

Hence by induction,

$$
h^{j}\left(x_{0}\right)=x_{j}+j b \quad \text { where } x_{j} \in E,
$$

and the $(n-1)$-planes $h^{j}(E)$ are pairwise disjoint for $j=0, \pm 1, \pm 2, \ldots$. Since $h-b$ is isotopic to the identity, we can now argue as in $[14$, p. 59] to obtain a self homeomorphism $g$ of $\mathbb{R}^{n}$ such that $h=g^{-1} \circ f \circ g$ where $f(x)=x+e_{1}$. 
Thus a quasitranslation is standard if and only if it is a topological translation and we obtain the following result.

7.30. THEOREM. When $n=2$, each orientation-preserving parabolic element $g$ is standard. For each $n \geqslant 3$ there exists an orientation-preserving non-standard parabolic element $g$.

7.31. THEOREM. For each $n \geqslant 2$ there exists a non-standard convergence group $G$ with exactly one point in $L(G)$. 7.30.

Proof. When $n \geqslant 3$, we can set $G=\langle g\rangle$ where $g$ is the element in Theorem

We give an alternative argument which includes the case where $n=2$. Let $H$ be a Möbius group with at least $n+1$ generators that contains only loxodromic elements which map the unit $n$-ball $B^{n}$ onto itself. Next let $f$ be a homeomorphism which maps $B^{n}$ onto $\mathbb{R}^{n}$ and set $G=f \circ H \circ f^{-1}$. Then each $g$ in $G$ has a homeomorphic extension $g^{*}$ to $\mathbb{R}^{n}$ and the corresponding group of extensions $G^{*}$ is a discrete convergence group all of whose elements are parabolic and fix $\infty$. However $G^{*}$ cannot be topologically conjugate to a Möbius group since each such group is, in turn, conjugate to a discrete group of euclidean isometries of $\mathbb{R}^{n}$ and hence can have at most $n$ generators of infinite order by, for example, [33, Theorem 3.2.8].

7.32. Loxodromic elements. If $g$ is loxodromic with $x_{0}=0$ and $y_{0}=\infty$ as its repulsive and attractive fixed points, then

$$
\lim _{j \rightarrow \infty} g^{j}=\infty \text { and } \lim _{j \rightarrow \infty} g^{-j}=0
$$

$c$-uniformly in $\overline{\mathbb{R}}^{n} \backslash\{0\}$ and $\mathbb{R}^{n}$, respectively. The orientation-preserving mappings $g$ which satisfy this condition are called topological dilations, and by results due to Kerékjártó [17], Homma and Kinoshita [13], and Husch [15], they are topologically conjugate to a mapping of the form $f(x)=2 x$ whenever $n=2,3$, or $n \geqslant 6$.

7.33. THEOREM. When $n=2,3$ or $n \geqslant 6$, each sense-preserving loxodromic element $g$ is standard.

Theorem 7.33 probably holds when $n=4,5$; the difficulty for these cases involves a problem in four-dimensional topology. In any case, Theorem 7.33 suggests that it may be more difficult to exhibit a non-standard discrete convergence group $G$ with $\operatorname{card}(L(G)) \geqslant 2$ than for the cases where $\operatorname{card}(L(G))=0$ and $\operatorname{card}(L(G))=1$.

When $n=3$, the existence of such a group $G$ with $\operatorname{card}(L(G))>2$ is an immediate consequence of the following important result due to Freedman and Skora [6].

THEOREM 7.34. For $n=3$ there exists a non-standard non-elementary discrete quasiconformal group $G$. 
In Part II of this article we shall use combination theorems to construct non-standard non-elementary discrete quasiconformal, and hence convergence, groups in $\overline{\mathbb{R}}^{n}$ for each $n>3$.

7.35. Lifting problem. An important tool for studying discrete groups of Möbius transformations acting on $\overline{\mathbb{R}}^{2}$ results from the fact that each such group $G$ can be extended to a Möbius group $G^{*}$ acting on $\overline{\mathbb{R}}^{3}$. Thus it is natural to ask when a discrete convergence group $G$ acting on $\overline{\mathbb{B}}^{n}$ can be extended to a discrete convergence group $G^{*}$ acting on $\mathbb{R}^{n+1}$. A recent result of Freedman [7] shows that this is possible for the case where $n \neq 3$ if $G$ is isomorphic to a free group with $k$ generators and $L(G)$ is a Cantor set. The case where $n=3$ is equivalent to the validity of 4-dimensional surgery [7].

\section{References}

1. S. AGARD, Elementary properties of Möbius transformations in $\mathbb{R}^{n}$ with applications to rigidity theory, University of Minnesota Mathematics Report 82-110.

2. A. F. BEARDON, The geometry of discrete groups (Springer, Berlin, 1983).

3. A. BEURLING and L. AhLFors, 'The boundary correspondence under quasiconformal mappings', Acta Math. 96 (1956) 125-142.

4. B. BojARSKI and T. IWANIEC, 'Another approach to Liouville theorem', Math. Nachr. 107 (1982) 253-262.

5. S. EILENBERG, 'Sur les transformations périodiques de la surface du sphère', Fund. Math. 22 (1934) 28-41.

6. M. H. FREEDMAN and R. SkORA, 'Strange actions of groups on spheres', J. Differential Geom. 25 (1987) 75-98.

7. M. H. Freedman, 'A geometric reformulation of 4-dimensional surgery', J. Top. Appl., to appear.

8. F. W. GeHRING, 'Rings and quasiconformal mappings in space', Trans. Amer. Math. Soc. 103 (1962) 353-393.

9. F. W. Gehring and B. P. Palka, 'Quasiconformally homogeneous domains', J. Analyse Math. 30 (1976) 172-199.

10. C. H. Giffen, 'The generalized Smith conjecture', Amer. J. Math. 88 (1966) 187-198.

11. A. HinkKanen, 'Uniformly quasisymmetric groups', Proc. London Math. Soc. 51 (1985) 318-338.

12. J. G. Hocking and G. S. Young, Topology (Addison-Wesley, Reading, Mass., 1961).

13. T. HommA and S. KINoshitA, 'On a topological characterization of the dilatation in $E^{3}$, Osaka Math. J. 6 (1954) 135-143.

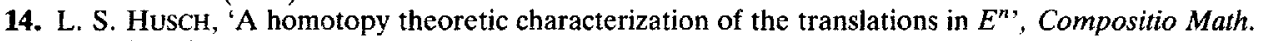
24 (1972) 55-61.

15. L. S. HusCH, 'A topological characterization of the dilation in $E^{n}$, Proc. Amer. Math. Soc. 28 (1971) 234-236.

16. B. von KeRÉKJÁRTó, 'Über die fixpunktfreien Abbildungen der Ebene', Acta Litt. Sci. Szeged 6 (1934) 226-234.

17. B. VON KERÉKJÁRTÓ, 'Topologische Charakterisierung der linearen Abbildungen', Acta Litt. Sci. Szeged 6 (1934) 235-262.

18. S. Kinoshita, 'On quasi-translation in 3-space', Fund. Math. 56 (1964) 69-79.

19. R. S. Kulkarni, 'Some topological aspects of Kleinian groups', Amer. J. Math. 100 (1976) 897-911.

20. W. J. LEVEQuE, Topics in number theory I (Addison-Wesley, Reading, Mass., 1958).

21. G. J. MARTIN, 'Discrete quasiconformal groups that are not the quasiconformal conjugates of Möbius groups', Ann. Acad. Sci. Fenn. 11 (1986).

22. M. J. M. MCKemIE, 'Quasiconformal groups and quasisymmetric embeddings', dissertation, University of Texas, 1985.

23. D. Montgomery and L. ZipPIN, 'Examples of transformation groups', Proc. Amer. Math. Soc. 5 (1954) 460-465.

24. J. W. MORGAN and H. BAss, The Smith conjecture (Academic Press, New York, 1984). 
25. M. H. A. Newman, 'A theorem on periodic transformations of spaces', Quart. J. Math. 2 (1931) $1-8$.

26. JU. G. RESHNETYAK, 'Liouville's theorem on conformal mappings under minimal regularity assumptions' (in Russian), Sibirsk. Math. Zh. 8 (1967) 835-840.

27. D. SULLIVAN, 'On the ergodic theory at infinity of an arbitrary discrete group of hyperbolic motions', Riemann surfaces and related topics: proceedings of the 1978 Stony Brook Conference, Annals of Mathematics Studies 97 (Princeton University Press, 1981), pp. 465-496.

28. P. Tukia, 'On two-dimensional groups', Ann. Acad. Sci. Fenn. 5 (1980) 73-78.

29. P. Tukia, 'A quasiconformal group not isomorphic to a Möbius group', Ann. Acad. Sci. Fenn. 6 (1981) 149-160.

30. P. TukiA and J. VÄISÄLÄ, 'A remark on 1-quasiconformal maps', Ann. Acad. Sci. Fenn. 10 (1985) 561-562.

31. J. VÄISÄLÄ, Lectures on n-dimensional quasiconformal mappings, Lecture Notes in Mathematics 229 (Springer, Berlin, 1971).

32. R. L. WILDER, Topology of manifolds (American Mathematical Society, Providence, R.I., 1949).

33. J. A. WOLF, Spaces of constant curvature (Publish or Perish, Wilmington, Delaware, 1984).

\author{
Department of Mathematics \\ University of Michigan \\ Ann Arbor \\ Michigan 48109-1003 \\ U.S.A.
}

\author{
Department of Mathematics \\ Yale University \\ New Haven \\ Connecticut 06520 \\ U.S.A.
}

